\title{
Micromechanical analysis of kinematic hardening in natural clay
}

\author{
Zhen-Yu Yin ${ }^{\mathrm{a}, \mathrm{b}, \mathrm{c}, \mathrm{d}, *}$, Ching S. Chang ${ }^{\mathrm{b}}$, Pierre-Yves Hicher ${ }^{\mathrm{c}}$, Minna Karstunen ${ }^{\mathrm{d}}$ \\ ${ }^{a}$ Department of Civil and Environmental Engineering, Helsinki University of Technology, PO Box 2100, 02015 TKK, Finland \\ ${ }^{\mathrm{b}}$ Department of Civil and Environmental Engineering, University of Massachusetts, Amherst, MA 01002, USA \\ ${ }^{\mathrm{c}}$ Research Institute in Civil and Mechanical Engineering, GeM UMR CNRS 6183, Ecole Centrale de Nantes, BP 92101, 44321 Nantes \\ Cedex 3, France \\ ${ }^{\mathrm{d}}$ Department of Civil Engineering, University of Strathclyde, John Anderson Building, 107 Rottenrow, Glasgow G4 0NG, UK
}

\begin{abstract}
A B S T R A C T
This paper presents a micromechanical analysis of the macroscopic behaviour of natural clay. A microstructural stress-strain model for clayey material has been developed which considers clay as a collection of clusters. The deformation of a representative volume of the material is generated by mobilizing and compressing all the clusters along their contact planes. Numerical simulations of multistage drained triaxial stress paths on Otaniemi clay have been performed and compared the numerical results to the experimental ones in order to validate the modelling approach. Then, the numerical results obtained at the microscopic level were analysed in order to explain the induced anisotropy observed in the clay behaviour at the macroscopic level. The evolution of the state variables at each contact plane during loading can explain the changes in shape and position in the stress space of the yield surface at the macroscopic level, as well as the rotation of the axes of anisotropy of the material.
\end{abstract}

\section{Introduction}

Natural soft clays often exhibit a significant degree of anisotropy, which is developed during their geological formation as in deposition, sedimentation, consolidation or by any subsequent straining (see, e.g., Tavenas and Lereoueil, 1977; Muir Wood, 1990; Burland, 1990; Diaz Rodriguez et al., 1992). 
In order to take into account the anisotropic mechanical behaviour of clay, a number of elastic-plastic stress-strain models have been developed, such as the models by Nova (1985), Dafalias (1986), Whittle and Kavvadas (1994), Pestana and Whittle (1999), Wheeler et al. (2003), Dafalias et al. (2006), etc. Hashiguchi and Mase (2007) developed a model employing a rotational kinematic hardening function for modelling the anisotropy of cemented sand. Yang et al. (2006) proposed a middle surface model using three pseudo-yield surfaces. A kinematic hardening rule was applied to the second pseudo-yield surface for modelling the anisotropic behaviour of sand. More recently, various kinematic hardening rules have been, respectively, investigated for frozen soils, unsaturated soils, and granular media by Lai et al. (2008), Muraleetharan et al.(2008), and Tsutsumi and Kaneko (2008). The key feature of these models is to adopt an asymmetrical yield surface for the modelling of inherent anisotropy due to the geological formation process, and to incorporate a kinematic hardening law for the modelling of induced anisotropy, in which the kinematic hardening law describes how the yield surface moves and changes its shape with the applied stresses. Both the initial yield surface and the kinematic hardening law of these models have been constructed phenomenologically from experimental results. Due to the complex nature of soil behaviour, it is difficult to construct a kinematic hardening law that is simple, effective, and at the same time, capable of capturing correctly the salient features of soil behaviour.

Besides the kinematic hardening approach, a potentially attractive way of modelling anisotropic material is the microstructural approach, in which the stress-strain relationship of a representative element is obtained by mobilizing contact planes of various orientations. The concept goes back to Taylor and Budiansky in their models for polycrystalline material (e.g., Batdorf and Budianski, 1949). Similar approaches can also be found in the models of rock and soils (e.g., Calladine, 1971; multilaminate models by Pande and Sharma, 1982; Cudny and Vermeer, 2004), in the models of concrete (e.g., micro-plane model by Bazant et al., 1995), and in the models of granular materials and sands (e.g., Chang and Liao, 1990; Chang and Gao, 1995; Chang and Hicher, 2005; Nicot and Darve, 2007).

The proposed approach can better model anisotropic material due to the following two reasons: (1) the state variables (local stress and strain) are naturally different in the contact planes according to their orientations related to the applied load. Since contact stiffness and contact strength are stressdependent, this would lead to different properties for each plane. Thus, the applied stress would create anisotropy for the material in a natural manner; (2) the evolution of the state variables (local stress and strain) is attained directly from the applied stress on each contact plane. There is no need to define a yield surface and a kinematic hardening rule in order to follow the evolution of the anisotropy.

In this paper, the development of a microstructure based elasto-plastic constitutive model is first presented. The model is then used to predict multistage drained triaxial stress path tests on Otaniemi clay. A numerical microstructural investigation is also carried out, which is intended to explain the induced anisotropy through the behaviour on contact planes. Finally, the microstructural model is used to construct the yield surface and to explain macro kinematic hardening of yield surface, i.e., how the yield surface expands, rotates, and changes its shape due to different stress paths.

\section{Constitutive model}

A clay particle is usually platy in shape. The size for a platy particle generally ranges from 0.01 to $1 \mu \mathrm{m}$ depending on the clay type (e.g., montmorillonite, illite or kaolinite). Clay particles attract each other due to surface forces among particles such as chemical, electrostatic, van der Waals forces, etc. These forces pull together the particles to form particle-clusters. The size of the clusters continues to grow until the clusters are large enough so that the cluster weight, due to gravitation, becomes significantly larger than the inter-particle surface forces. At this stage, the cluster looses its potential to attract further clay particles, and the size of clusters stops to grow. The ultimate cluster size depends on the clay particle type, the liquid inside the pores, and its sedimentation history.

From the photos of clay material under scanning electron microscopes, clusters formed by platy clay particles can be identified as rotund shape, although the microfabric within a cluster may be either a flocculate or dispersed type structure (Hicher et al., 2000).

At the size of clusters, long range forces such as electrostatic and van der Waals forces are negligible, and clusters interact with each other mainly through mechanical forces. Thus, clay material, 
considered as a collection of clusters, can be modelled by analogy to granular material. This explains why sand and clay have similar qualitative behaviour even though each material consists of different constituents (Biarez and Hicher, 1994).

The present model is extended from the sand model developed by Chang and Hicher (2005). In this model, clay is envisioned as an aggregate of clusters. The deformation of a representative volume of the material is generated by mobilizing and compressing all clusters. Thus, the stress-strain relationship can be derived as an average of the deformation behaviour of local contact planes in all orientations. For contact planes in the $\alpha$ th orientation, the local forces $f_{j}^{\alpha}$ and the local movements $\delta_{i}^{\alpha}$ can be denoted as follows: $f_{j}^{\alpha}=\left\{f_{n}^{\alpha}, f_{s}^{\alpha}, f_{t}^{\alpha}\right\}$ and $\delta_{i}^{\alpha}=\left\{\delta_{n}^{\alpha}, \delta_{s}^{\alpha}, \delta_{t}^{\alpha}\right\}$, where the subscripts $n, s$, and $t$ represent the components in the three directions of the local coordinate system as shown in Fig. 1. The direction outward normal to the plane is denoted as $n$; the other two orthogonal directions, $s$ and $t$, are tangential to the plane.

\subsection{Density state of clay}

One of the important elements to consider in modelling clay behaviour is the critical state concept. At critical state, the clay material remains at constant volume while it is subjected to a continuous distortion. The void ratio corresponding to this state is termed critical void $e_{c}$, which is a function of the effective mean stress $p=\left(\sigma_{x}+\sigma_{y}+\sigma_{z}\right) / 3$ (all stress terms used in the part of constitutive model refer to effective stress). The relationship has traditionally been written as follows:

$$
e_{c}=e_{c 0}-\lambda \ln \left(\frac{p}{p_{c r 0}}\right)
$$

The two parameters $\left(e_{c 0}, p_{c r 0}\right)$ represent a reference point on the critical state line. For convenience, the value of $p_{c r 0}$ is taken to be $1 \mathrm{kPa}$. The critical state line can be defined by two parameters $e_{c 0}$ and $\lambda$. Using the critical state concept, the density state of an assembly under a given mean effective stress is defined as the ratio $e / e_{c}$, where $e$ is the void ratio of the assembly and $e_{c}$ the critical void ratio at the same given stress state.

The relationship between void ratio and isotropic stress in semi-log scale $(e-\log p)$ is assumed to be linear. However, some investigators prefer to use a linear relationship between $\log \varepsilon_{v}-\log p$ for clay with large deformation (Hashiguchi, 2008).

\subsection{Inter-cluster behaviour}

Since contact forces and applied stresses have different units, it is troublesome to compare their magnitudes. Thus, local stresses and local strains are introduced for convenience. We define a local stress $\tau_{i}^{\alpha}$ and a local strain $\gamma_{i}^{\alpha}$, which are directly related to the local force $f_{j}^{\alpha}$ and the local movement $\delta_{i}^{\alpha}$ at each contact, given by

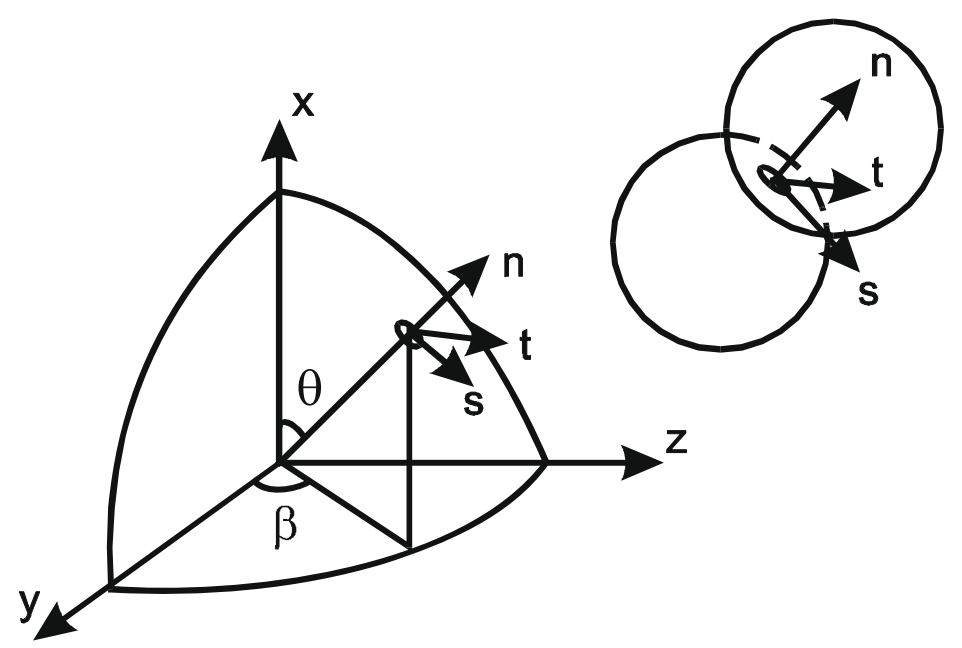

Fig. 1. Local coordinate at inter-particle contact. 


$$
\tau_{i}^{\alpha}=\frac{N l^{\alpha}}{3 V} f_{i}^{\alpha} ; \quad \gamma_{i}^{\alpha}=\delta_{i}^{\alpha} / l^{\alpha}
$$

where $l^{\alpha}$ is the length of the branch vector, which joins the centroids of two contacting clusters. $V$ is the volume of the representative element. $N$ is the total number of contacts. The form of the local stress is derived from the static hypothesis given by Liao et al. (1997)

$$
\dot{f}_{j}^{\alpha}=\dot{\sigma}_{i j} A_{i k} l_{k}^{\alpha}
$$

where $A_{i k}$ is the inverse of fabric tensor $A_{i k}=\left[\frac{1}{V} \sum_{\alpha=1}^{N} l_{i}^{\alpha} l_{k}^{\alpha}\right]^{-1}$ For the case of an isotropic fabric, it can be derived that $A_{i k}=3 V /\left(N l^{2}\right) \delta_{i k}$, where $\delta_{i k}$ is the Kronecker delta. In this case, Eq. (3) implies $\sigma_{j i} n_{j}^{\alpha}=N l^{\alpha} /(3 V) f_{i}^{\alpha}$. Therefore, the local stress defined in Eq. (2) is equal in magnitude to the traction resolved from the applied stress on the contact plane (i.e., $\tau_{i}^{\alpha}=\sigma_{j i} n_{j}^{\alpha}$ ) for an isotropic packing structure. It is to be noted that the local stress $\tau_{i}^{\alpha}$ is not the true stress on the physical contact area between the two clusters. It should be rather viewed as a normalized form of the contact force.

In the local coordinate system, the local stress and local strain are, respectively, denoted as $\left\{\begin{array}{lll}\tau_{n}^{\alpha} & \tau_{s}^{\alpha} & \tau_{t}^{\alpha}\end{array}\right\}$ and $\left\{\begin{array}{lll}\gamma_{n}^{\alpha} & \gamma_{s}^{\alpha} & \gamma_{t}^{\alpha}\end{array}\right\}$. For convenience, we use the notation $\sigma^{\alpha}=\tau_{n}^{\alpha}$ for local normal stress and the notation $\varepsilon^{\alpha}=\gamma_{n}^{\alpha}$ for local normal strain in the following sections.

\subsubsection{Elastic part}

The inter-cluster behaviour can be characterized as the relationship between local stress and local strain, given by

$$
\tau_{i}^{\alpha}=\bar{k}_{i j}^{\alpha} \gamma_{j}^{\alpha}
$$

in which the stiffness tensor can be related to the contact normal stiffness, $\bar{k}_{n}^{\alpha}$, and shear stiffness, $\bar{k}_{r}^{\alpha}$,

$$
\bar{k}_{i j}^{\alpha}=\bar{k}_{n}^{\alpha} n_{i}^{\alpha} n_{j}^{\alpha}+\bar{k}_{r}^{\alpha}\left(s_{i}^{\alpha} s_{j}^{\alpha}+t_{i}^{\alpha} t_{j}^{\alpha}\right)
$$

The inter-cluster stiffness can be expressed as the form adopted for sand grains by Chang et al. (1989), given by

$$
\bar{k}_{n}^{\alpha}=\bar{k}_{n 0}^{\alpha}\left(\frac{\sigma^{\alpha}}{p_{\text {ref }}}\right)^{n} ; \quad \bar{k}_{r}^{\alpha}=k_{r R} \bar{k}_{n}^{\alpha}=k_{r R} \bar{k}_{n 0}^{\alpha}\left(\frac{\sigma^{\alpha}}{p_{\text {ref }}}\right)^{n}
$$

where $\sigma^{\alpha}$ is the local stress in normal direction, $p_{\text {ref }}$ is the standard reference pressure taken as $1 \mathrm{kPa}$, and $k_{r R}$ is the ratio of shear to normal stiffness. $\bar{k}_{n 0}^{\alpha}, k_{r R}$ and $n$ are material constants. The value of $n$ is found to be 0.33 for two elastic spheres according to Hertz-Mindlin's formulation (1969). Based on experimental measurements of elastic modulus under different confining stress, the value of $n$ has been found to be $0.5-1.0$ for clay.

\subsubsection{Plastic part}

2.2.2.1. Shear sliding. Plastic sliding often occurs along the tangential direction of the contact plane with an upward or downward movement (i.e., dilation or contraction). The dilatancy equation used here is modified from the equation adopted for sand by Chang and Hicher (2005), given by

$$
\frac{d \varepsilon^{p}}{d \gamma^{p}}=b\left(\frac{\tau}{\sigma}-\tan \phi_{\mu}\right)\left(\frac{\tau}{\sigma}\right)^{a}\left(1-\frac{e}{e_{c}}\right)
$$

The modified equation allows more flexibility in modelling the performance of different material behaviour. In this equation, $a, b$, and $\phi_{\mu}$ are inter-cluster property constants; $e_{c}$ is the critical void ratio for the clay. When the void ratio $e$ is equal to the critical void ratio, zero dilation holds. It is noted that the state variables $e$ and $e_{c}$ of the clay are at a macro-scale of the cluster assembly, which is used to regulate the dilation of individual inter-cluster contacts. It is reasonable to consider the micro variable as a function of the macro-state, because the inter-cluster behaviour is indeed influenced by the density state of the specimen.

In Eq. (7), $\phi_{\mu}$ is the inter-cluster friction angle, which in value is very close to the internal friction angle measured at critical state. The values of $a$, and $b$ can be calibrated from experimental measurements of triaxial tests, which will be shown in the later section on numerical simulation. 
Note that the shear stress $\tau$ and the rate of plastic shear strain $d \gamma^{p}$ in Eq. (7) are defined as

$$
\tau=\sqrt{\tau_{s}^{2}+\tau_{t}^{2}} \text { and } d \gamma^{p}=\sqrt{\left(d \gamma_{s}^{p}\right)^{2}+\left(d \gamma_{t}^{p}\right)^{2}}
$$

The yield function is assumed to be of Mohr-Coulomb type, given by

$$
F_{1}\left(\tau, \sigma, \kappa_{1}\right)=\tau-\sigma \kappa_{1}\left(\gamma^{p}\right)=0
$$

where $\kappa_{1}\left(\gamma^{p}\right)$ is an isotropic hardening/softening parameter. The hardening parameter is defined by a hyperbolic function in the $\kappa_{1}-\gamma^{p}$ plane, which involves two material constants: $\phi_{p}$ and $\bar{k}_{p}$.

$$
\kappa_{1}=\frac{\bar{k}_{p} \tan \phi_{p} \gamma^{p}}{\sigma \tan \phi_{p}+\bar{k}_{p} \gamma^{p}}
$$

When plastic deformation increases, $\kappa_{1}$ approaches asymptotically $\tan \phi_{p}$. For a given value of $\sigma$, the initial slope of the hyperbolic curve is $\bar{k}_{p} / \sigma$. Under a loading condition, the shear plastic flow in the direction tangential to the contact plane is determined by a normality rule applied to the yield function. However, the plastic flow in the direction normal to the contact plane is governed by the stress-dilatancy equation in Eq. (7). Therefore, the flow rule is non-associated.

The value of $\bar{k}_{p}$ is found to be linearly proportional to $\bar{k}_{n}$ so that

$$
\bar{k}_{p}^{\alpha}=k_{p R} \bar{k}_{n}^{\alpha}=k_{p R} \bar{k}_{n 0}^{\alpha}\left(\frac{\sigma^{\alpha}}{p_{\text {ref }}}\right)^{n}
$$

The ratio $k_{p R}$ is a material parameter.

The internal friction angle $\phi_{\mu}$ is a constant for a given material. However, the peak friction angle, $\phi_{p}$, on a contact plane is dependent on the density state of neighbouring clusters, which can be related to the void ratio $e$ by

$$
\tan \phi_{p}=\left(\frac{e_{c}}{e}\right)^{m} \tan \phi_{\mu}
$$

where $m$ is a material constant (Biarez and Hicher, 1994).

In a loose structure, clusters can rotate more freely, preventing the inter-cluster shear force from fully mobilizing the sliding resistance. The peak frictional angle $\phi_{p}$ is smaller than $\phi_{\mu}$. On the other hand, a dense structure provides a higher degree of interlocking, which requires more effort to mobilize the clusters in contact. In this case, the peak frictional angle $\phi_{p}$ is greater than $\phi_{\mu}$. When the dense structure starts to dilate, the degree of interlocking relaxes. As a consequence, the peak frictional angle is reduced, which results in a strain-softening phenomenon.

2.2.2.2. Normal compression. In order to describe the compressible behaviour between two clay clusters, a second yield function is hence added. The second yield function is assumed to be as follows:

$$
F_{2}\left(\sigma, \kappa_{2}\right)=\sigma-\kappa_{2}\left(\varepsilon^{p}\right) \text { for } \sigma>\sigma_{p}
$$

where the local normal stress $\sigma$ and local normal strain $\varepsilon^{p}$ are defined in Eq. (3). In analogy to the macro volume compression behaviour, we express the hardening function $\kappa_{2}\left(\varepsilon^{p}\right)$ in a semi-logarithmic form given by

$$
\kappa_{2}=\sigma_{p} 10^{\varepsilon^{p} / c_{p}} \quad \text { or } \quad \varepsilon^{p}=c_{p} \log \frac{\kappa_{2}}{\sigma_{p}}
$$

where $c_{p}$ is the compression index for the compression curve plotted in the $\varepsilon^{p}-\log \sigma$ plane. When the compression $\sigma$ is less than $\sigma_{p}$, the plastic strain produced by the second yield function is null. Thus, $\sigma_{p}$ in Eq. (12) corresponds to the pre-consolidation stress in soil mechanics.

\subsubsection{Elasto-plastic relationship}

With the basic elements of inter-cluster behaviour discussed above, the final incremental local stress-strain relation of the inter-cluster contact can be derived, including both elastic and plastic behaviour, given by 


$$
\dot{\tau}_{i}^{\alpha}=\bar{k}_{i j}^{\alpha p} \dot{\gamma}_{j}^{\alpha}
$$

Since detailed derivation of the elasto-plastic stiffness tensor is standard, it will not be given here.

\subsection{Stress-strain relationship}

\subsubsection{Macro-micro relationship}

The stress-strain relationship for an assembly of clay clusters can be determined from integrating the inter-cluster behaviour at all contacts. During the integration process, a relationship is required to link the macro and micro variables.

In a micromechanical expression, following the Love-Weber formula, the stress increment $\dot{\sigma}_{i j}$ can be obtained by adding the diatic product of the contact force and the branch vectors for all contacts (Christofferson et al., 1981; Rothenburg and Selvadurai, 1981). In terms of local stress, it is

$$
\dot{\sigma}_{i j}=\frac{1}{V} \sum_{\alpha=1}^{N} f_{j}^{\alpha} l_{i}^{\alpha}=\frac{3}{N} \sum_{\alpha=1}^{N} \tau_{j}^{\alpha} n_{i}^{\alpha}
$$

In terms of the local stress on the $\alpha$ th contact plane defined in Eq. (2), the static hypothesis of Eq. (3) becomes

$$
\dot{\tau}_{j}^{\alpha}=\dot{\sigma}_{i j} B_{i k}^{\alpha} n_{k}^{\alpha}
$$

where the tensor $B_{i k}^{\alpha}$ in Eq. (17) is defined as $B_{i k}^{\alpha}=N /(3 V) A_{i k}\left(l^{\alpha}\right)^{2}$.

Using the principle of energy balance, which states that the work done in a representative volume element is equal to the work done on all inter-cluster planes within the element,

$$
\sigma_{i j} \dot{u}_{j, i}=\frac{1}{V} \sum_{\alpha=1}^{N} f_{j}^{\alpha} \dot{\delta}_{j}^{\alpha}=\frac{3}{N} \sum_{\alpha=1}^{N} \tau_{j}^{\alpha} \dot{\gamma}_{j}^{\alpha},
$$

Substituting the local stress in Eq. (17) into Eq. (18), the relationship between the strain of assembly and inter-cluster strain is obtained

$$
\dot{u}_{j, i}=\frac{3}{N} \sum_{\alpha=1}^{N} \dot{\gamma}_{j}^{\alpha} n_{k}^{\alpha} B_{i k}^{\alpha}
$$

where $\dot{\gamma}_{j}$ is the local strain between two contact clusters, $n_{k}$ the unit vector of the branch joining the centres of two contact clusters, and $N$ the total number of contacts, over which the summation is carried out.

Using Eqs. (15), (19), and (17), the following relationship between stress and strain can be obtained:

$$
\dot{u}_{i, j}=C_{i j m p} \dot{\sigma}_{m p}
$$

where

$$
C_{i j m p}=\frac{3}{N} \sum_{\alpha=1}^{N}\left(\bar{k}_{j p}^{e p}\right)^{-1} n_{k}^{\alpha} n_{n}^{\alpha} B_{i k}^{\alpha} B_{m n}^{\alpha}
$$

The summation in Eq. (21) can be expressed by a closed-form solution for some limited conditions such as the elastic modulus of randomly packed equal-size particles (Chang and Gao, 1995). However, in an elastic-plastic behaviour, due to the nonlinear nature of the local constitutive equation, a numerical calculation with an iterative process is necessary to carry out the summation in Eq. (21) (see Chang and Hicher, 2005).

\subsection{Summary of parameters}

The material parameters are summarised as follows:

(1) Microstructural descriptions (two parameters)

- Contact number per unit volume, $N / V$ and mean cluster size, $d$ 
(2) Inter-cluster properties (nine parameters)

- Inter-cluster elastic constants: $\bar{k}_{n 0}, k_{r R}$, and $n$;

- Inter-cluster friction angle: $\phi_{\mu}$ and $m$;

- Inter-cluster plastic compression index and plastic shear stiffness ratio: $c_{p}$ and $k_{p R}$;

- Dilation constants: $a$ and $b$

(3) Density state of the assembly (three parameters)

- Critical state for the soil: $\lambda$ and $e_{c 0}$

- Reference void ratio, $e_{0}$, on the isotropic compression line at $p=0.001 \mathrm{MPa}$.

The size of a clay cluster $d$ can be estimated from an electron microscopic scanning photograph. The value of $N / V$ is not easy to obtain directly from the clay experiments. According to the experimental data by Oda (1977) for three mixtures of spheres, the contact number per unit volume can be approximately related to the void ratio by

$$
\bar{V}=\frac{12}{\pi d^{3}(1+e) e}
$$

Here we use this equation as a first-order approximation to estimate $N / V$ for clay by treating $d$ as the mean size of the clay clusters. It is noted that the value of contact number per unit volume changes with void ratio. The evolution is accounted for during the deformation process.

The mean size $d$ of the clay clusters is assumed to be $4 \mu \mathrm{m}$ according to the observations from Scanning Electronic Microscope (SEM) results on kaolinite (Hicher et al., 2000). The exponent $n$ is generally between 0.7 and 1.0 for clay, and a typical value of exponent $m$ is 1 . From an isotropic compression test, four parameters can be determined; namely, $e_{0}, \lambda, \bar{k}_{n 0}^{\alpha}$, and $c_{p}$. The void ratio $e_{0}$ and $\lambda$ can be measured directly from the compression line. The values of $\bar{k}_{n 0}^{\alpha}$ and $c_{p}$ can be calibrated from the slopes of the compression and rebound curves. The other parameters $\phi_{\mu}, k_{p R}, k_{r R}, a, b$, and $e_{c 0}$ can be obtained from drained triaxial tests (as shown later).

\section{Test simulation and analysis}

\subsection{Review of experimental results}

The anisotropic behaviour of a natural clay, Otaniemi clay in southern Finland, is presented herein with reference to experimental results based on the work of Wheeler et al. (2003) and Karstunen and Koskinen (2004). Otaniemi clay is classified as a high-plasticity clay with the following mineralogical compositions: quartz $23 \%$, feldspar $46 \%$, illite $15 \%$, chlorite $10 \%$, and kaolinite $5 \%$, determined at ETH Zurich by Messerklinger et al. (2003). The tests on Otaniemi clay were all performed on samples taken at depths of 3.4-4.7 m. Some physical properties of Otaniemi clay at this depth are presented in Table 1. Wheeler et al. (2003) indicated that there is a noticeable natural variation within this $1.2 \mathrm{~m}$ thick clay layer.

To investigate the induced anisotropic behaviour and the resulting kinematic hardening of the yield surface, 26 drained triaxial tests with different effective stress paths were selected ( 20 of them can be found in Wheeler et al., 2003). All tests were conducted using conventional triaxial cells with deviatoric force applied by dead weight loading for both compression and extension. Stress increments were generally applied at daily intervals with the increment size depending on the requirements of the individual test stage. Each test consists of two loading stages (see Fig. 2):

(1) At the first loading stage, the specimen is loaded in a drained condition at a constant value of $\eta_{1}$ to a final stress state $\left(p_{1}^{\prime}, q_{1}\right)$, and then unloaded with $\eta_{1}$ unchanged (where $\eta=q / p^{\prime}, q=\sigma_{1}-\sigma_{3}$, and $p^{\prime}=\left(\sigma_{1}^{\prime}+2 \sigma_{3}^{\prime}\right) / 3$ for triaxial condition, with positive and negative $\eta$ representing compression and extension tests, respectively). 
Table 1

Physical properties of Otaniemi clay of depth 3.4-4.7 m (after Wheeler et al., 2003).

\begin{tabular}{lc}
\hline Index property & Value \\
\hline Water content, $w(\%)$ & $85-130$ \\
Liquid limit, $w_{\mathrm{l}}(\%)$ & $80-111$ \\
Plastic limit, $w_{\mathrm{p}}(\%)$ & $26-29$ \\
Plasticity index, $I_{\mathrm{p}}$ & $54-82$ \\
Percentage of particles $<0.002 \mathrm{~mm}, \mathrm{C} 1 \%$ & $65-83$ \\
Organic content, Hm $(\%)$ & $0-0.7$ \\
Specific gravity, Gs & $2.76-2.80$ \\
Undrained shear strength, cu (kPa) & $6-9$ \\
Sensitivity, St & $7-14$ \\
\hline
\end{tabular}

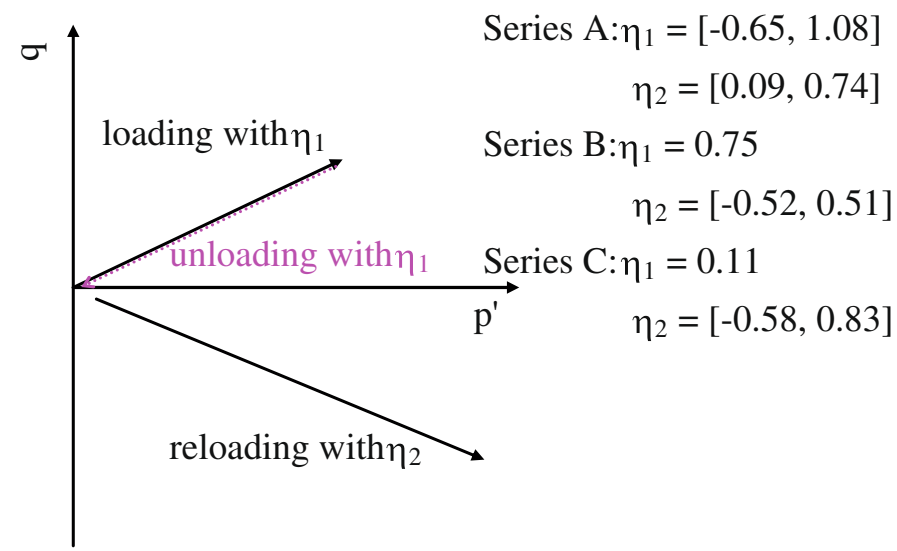

Fig. 2. Schematic description for test Series A, B, and C.

(2) At the second loading stage, the specimen is again loaded in a drained condition at a different constant value of $\eta_{2}$ to another final stress state $\left(p_{2}^{\prime}, q_{2}\right)$. All tests were classified in three series of tests (see Table 2, Wheeler et al., 2003):

- Series A: The samples were first loaded at various values of $\eta_{1}$ (ranging from -0.65 to 1.08 ) to a given stress state, and then each sample was loaded at a suitable alternative value of $\eta_{2}$ varying from 0.09 to 0.74 .

- Series B: The samples were first loaded at $\eta_{1}=0.75$ to $p_{1}^{\prime}=40 \mathrm{kPa}$, and then each sample was loaded at a different value of $\eta_{2}$ varying from -0.52 to 0.51 . The clay is subjected in situ to a value of $\eta$ about 0.75 .

- Series C: The samples were first loaded at $\eta_{1}=0.11$ to $p_{1}^{\prime}=45 \mathrm{kPa}$, and then at the second stage, each of the samples was loaded at values of $\eta_{2}$ varying from -0.58 to 0.83 .

For Series B and C, the specimens were consolidated up to $40-45 \mathrm{kPa}$ at the end of the first loading stage. The stress is higher than the in situ overburden pressure, which helps to reduce the sample's non-homogeneities due to the sampling process.

\subsection{Calibration of model parameters}

To calibrate the model parameters, we selected two experimental tests: (1) isotropic consolidation test (CID2241 listed in Table 2), and (2) an anisotropic consolidation test followed by an undrained triaxial compression (CAUC2239). The calibrated list of parameters is given in Table 3a. The parameters are calibrated based on the following process.

Parameter $\lambda=0.46$ was determined from the slope of the experimental consolidation curve (see Fig. 3a). The inter-cluster friction angle $\phi_{\mu}$ is determined from the slope of the critical state line in $p^{\prime}-q$ plane. A value of $\phi_{\mu}=30^{\circ}$ was determined from an undrained triaxial test (see Fig. 3b). A typical value of $m=1$ is used. The value of $p_{c r 0}$ and $e_{c 0}$ can be determined from the state (stress and void ratio) 
Table 2

Drained triaxial tests on Otaniemi clay.

\begin{tabular}{|c|c|c|c|c|c|c|c|c|c|c|}
\hline \multicolumn{2}{|c|}{ Test number } & \multirow[t]{2}{*}{ Depth (m) } & \multirow[t]{2}{*}{$w(\%)$} & \multirow[t]{2}{*}{$e_{i}$} & \multicolumn{3}{|c|}{ First loading } & \multicolumn{3}{|c|}{ Second loading } \\
\hline & & & & & $\eta_{1}$ & $p_{1}^{\prime}(\mathrm{kPa})$ & $q_{1}(\mathrm{kPa})$ & $\eta_{2}$ & $p_{2}^{\prime}(\mathrm{kPa})$ & $q_{2}(\mathrm{kPa})$ \\
\hline \multirow[t]{17}{*}{ Series A } & CAD2260 & $4.03-4.14$ & 97.8 & 2.73 & 1.08 & 33 & 32.4 & 0.1 & 150 & 15.3 \\
\hline & CAD2463 & $3.47-3.59$ & 119 & 3.43 & 1 & 37 & 37 & 0.3 & 100 & 30.3 \\
\hline & CAD2464 & $3.64-3.76$ & 114.9 & 3.26 & 0.89 & 38 & 33.8 & 0.33 & 101 & 33.8 \\
\hline & CAD2261 & $4.03-4.14$ & 92.5 & 2.62 & 0.79 & 37 & 29.2 & 0.1 & 150 & 15.4 \\
\hline & CAD2530 & $4.03-4.14$ & 101.4 & 3.1 & 0.6 & 40 & 20.32 & 0.32 & 71 & 22.5 \\
\hline & CAD2251 & $4.20-4.31$ & 90 & 2.51 & 0.6 & 40 & 24.1 & 0.09 & 150 & 14.1 \\
\hline & CAD2280 & $4.37-4.48$ & 93.6 & 2.54 & 0.51 & 50 & 25.4 & 0.1 & 120 & 12.5 \\
\hline & CAE2586 & $3.62-3.73$ & 112.4 & 3.23 & 0.43 & 44 & -18.8 & 0.51 & 96 & 49.2 \\
\hline & CAD2276 & $4.56-4.68$ & 79.4 & 2.59 & 0.26 & 48 & 12.4 & 0.11 & 150 & 15.9 \\
\hline & CAD2514 & $4.23-4.34$ & 85.4 & 2.98 & 0.21 & 36 & 7.5 & 0.74 & 60 & 44.5 \\
\hline & CAE2496 & $4.20-4.31$ & 93.5 & 2.54 & -0.34 & 31 & -10.5 & 0.1 & 66 & 6.7 \\
\hline & CAE2544 & $4.37-4.48$ & 88.1 & 2.98 & -0.6 & 33 & -19.8 & 0.51 & 66 & 33.7 \\
\hline & CAE2513 & $4.03-4.14$ & 104.7 & 2.91 & -0.65 & 29 & -18.8 & 0.61 & 60 & 36.8 \\
\hline & CID2241 & $4.02-4.14$ & 105 & 2.81 & 0 & 151 & 0 & 0.11 & 201 & 22.5 \\
\hline & CID2515 & $4.40-4.51$ & 92 & 2.2 & 0 & 60 & 0 & 0.6 & 100 & 60 \\
\hline & CID2291 & $4.37-4.48$ & 100.4 & 2.86 & 0 & 37 & 0 & 0.1 & 116 & 12 \\
\hline & CID2403 & $3.62-3.73$ & 114 & 3.3 & 0 & 42 & 0 & 0.42 & 99 & 42 \\
\hline \multirow[t]{4}{*}{ Series B } & CAD2443 & $4.23-4.34$ & 89.1 & 2.56 & 0.75 & 40 & 30 & 0.51 & 100 & 51 \\
\hline & CAD2425 & $4.06-4.17$ & 101.5 & 3.01 & & & & 0.22 & 97 & 21 \\
\hline & CAE2529 & $4.37-4.48$ & 84.1 & 2.50 & & & & -0.26 & 78 & -20 \\
\hline & CAE2522 & $4.12-4.23$ & 101.1 & 2.74 & & & & -0.52 & 60 & -31 \\
\hline \multirow[t]{5}{*}{ Series C } & CAD2424 & $4.03-4.14$ & 108.5 & 3.10 & & & & 0.83 & 60 & 50 \\
\hline & CAD2423 & $4.20-4.31$ & 94.4 & 2.61 & & & & 0.58 & 99 & 57 \\
\hline & CAD2422 & $4.05-4.17$ & 102.8 & 2.93 & 0.11 & 45 & 5 & 0.11 & 100 & 36 \\
\hline & CAE2561 & $3.95-4.06$ & 101.1 & 2.93 & & & & -0.45 & 87 & -39 \\
\hline & CAE2550 & $4.32-4.43$ & 90.8 & 2.63 & & & & -0.58 & 84 & -49 \\
\hline
\end{tabular}

Table 3

Values of model parameters for Otaniemi clay.

\begin{tabular}{|c|c|c|c|c|c|c|c|c|c|}
\hline \multirow[t]{2}{*}{ Parameters } & \multicolumn{4}{|c|}{ Global parameters } & \multicolumn{5}{|c|}{ Inter-particle parameters } \\
\hline & $e_{0}$ & $\lambda$ & $e_{c 0}$ & $\sigma_{p 0}^{\prime}(\mathrm{MPa})$ & $C_{p}$ & $\phi_{\mu}^{\prime}\left(^{\circ}\right)$ & $\bar{k}_{n 0}(\mathrm{MPa})$ & $k_{r R}$ & $k_{p R}$ \\
\hline a. CID2241 & 3.95 & 0.460 & 3.64 & 0.02 & 0.064 & 30 & 300 & 0.5 & 0.2 \\
\hline b. CID2291 & 4.36 & 0.595 & 3.91 & 0.021 & 0.08 & 30 & 380 & 0.5 & 0.2 \\
\hline c. CID2403 & 5.34 & 0.764 & 4.78 & 0.023 & 0.089 & 30 & 500 & 0.5 & 0.2 \\
\hline d. CID2515 & 3.43 & 0.437 & 3.12 & 0.03 & 0.068 & 30 & 290 & 0.5 & 0.2 \\
\hline Average & 4.27 & 0.564 & 3.86 & 0.0235 & 0.075 & 30 & 368 & 0.5 & 0.2 \\
\hline
\end{tabular}

corresponding to the critical state. The values of $p_{\text {ref }}$ and $e_{0}$ can be determined by an isotropic line, where $p_{\text {ref }}$ is $1 \mathrm{kPa}$ with the point $\left(p_{\text {ref }}, e_{0}\right)$ appearing on the isotropic line (see Fig. 3a). Fig. 3c shows a slight influence of $k_{r R}$ and $k_{p R}$ on simulating the isotropic consolidation test which means $k_{r R}$ and $k_{p R}$ are not sensitive for IC test. Other parameters can be obtained by curve fitting, as shown in Fig. 3d-i, as follows:

(1) Inter-cluster elastic constants: $\bar{k}_{n 0}, k_{r R}$ and $n$;

The exponent $n=1$ was considered which provides a linear $\kappa$-line (unloading-reloading curve in $e-\log p^{\prime}$ of the consolidation test). The value of $\bar{k}_{n 0}$ was determined from the $\kappa$-line, as shown in Fig. $3 \mathrm{~d}$. $k_{r R}$ was determined from the $\varepsilon_{v}-\varepsilon_{1}$ (volumetric strain versus axial strain) curve of the isotropic consolidation test (see Fig. 3e).

(1) Inter-cluster normal hardening rule: $c_{p}$ and $\sigma_{p 0}^{\prime}$; 

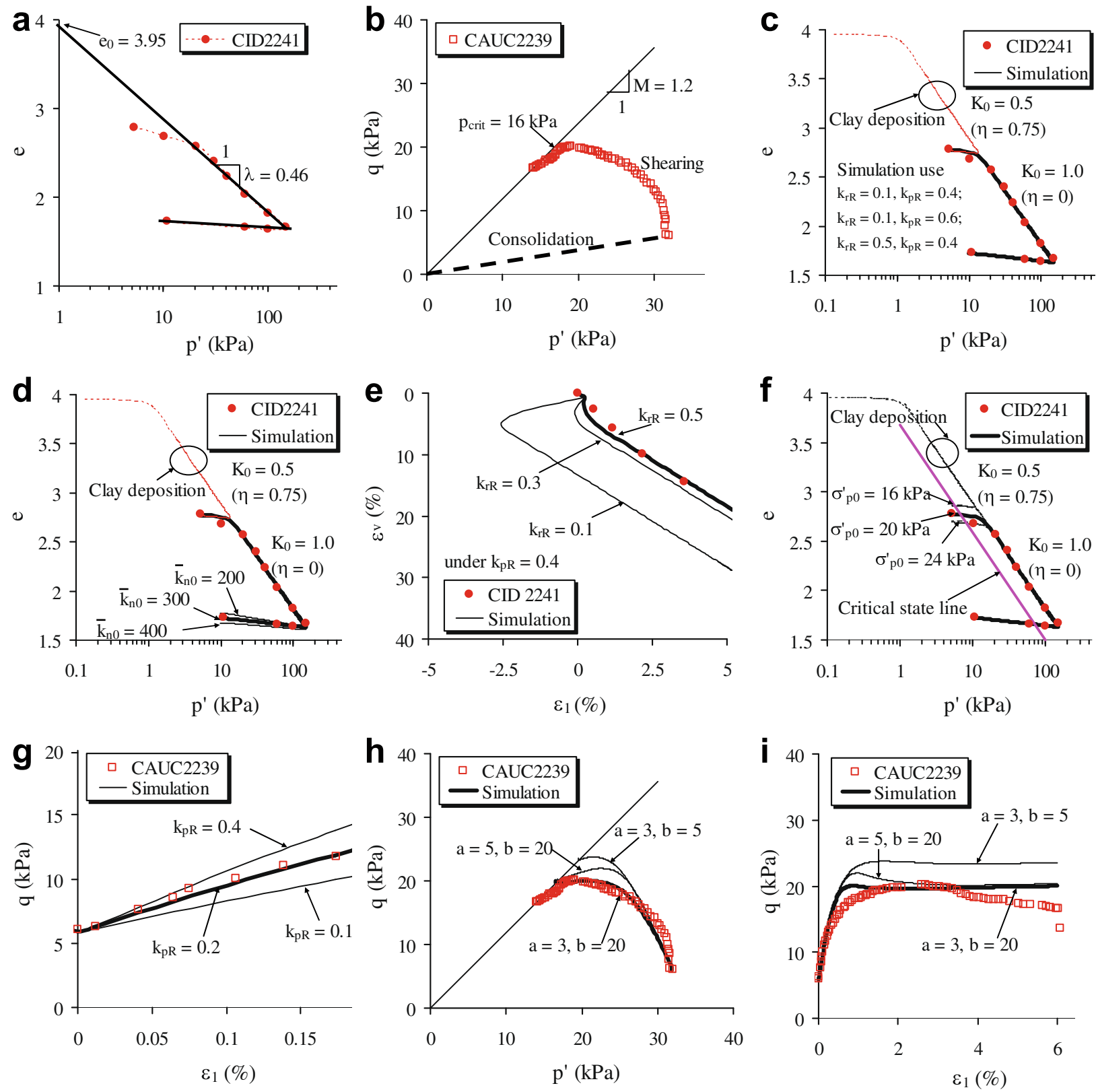

Fig. 3. Calibration of model parameters from isotropic consolidation test and undrained triaxial test.

The value of $c_{p}$ was determined by keeping the isotropic consolidation line parallel to the critical state line (see Fig. 3f). The initial value of the pre-consolidation pressure $\sigma_{p 0}^{\prime}$ due to the clay deposition history was determined, as shown in Fig. 3f.

(1) Inter-cluster shear hardening rule: $k_{p R}$;

The value of $k_{p R}$ was determined from the $q-\varepsilon_{1}$ curve of an undrained compression test at small strain, as shown in Fig. 3g.

(1) Dilation constants $a$ and $b$ were determined from an undrained compression test (see Fig. 3h and i); from this curve, combined values of $a$ and $b$ can be chosen by curve fitting. Parameters $a$ and $b$ governing the amount of volumetric dilation have a significant influence on the peak strength of the $q-\varepsilon_{1}$ curve. Fig. 3h shows that different effective stress paths, with a similar shape of the yield curve, can be described by different values of $a$ and $b$. 

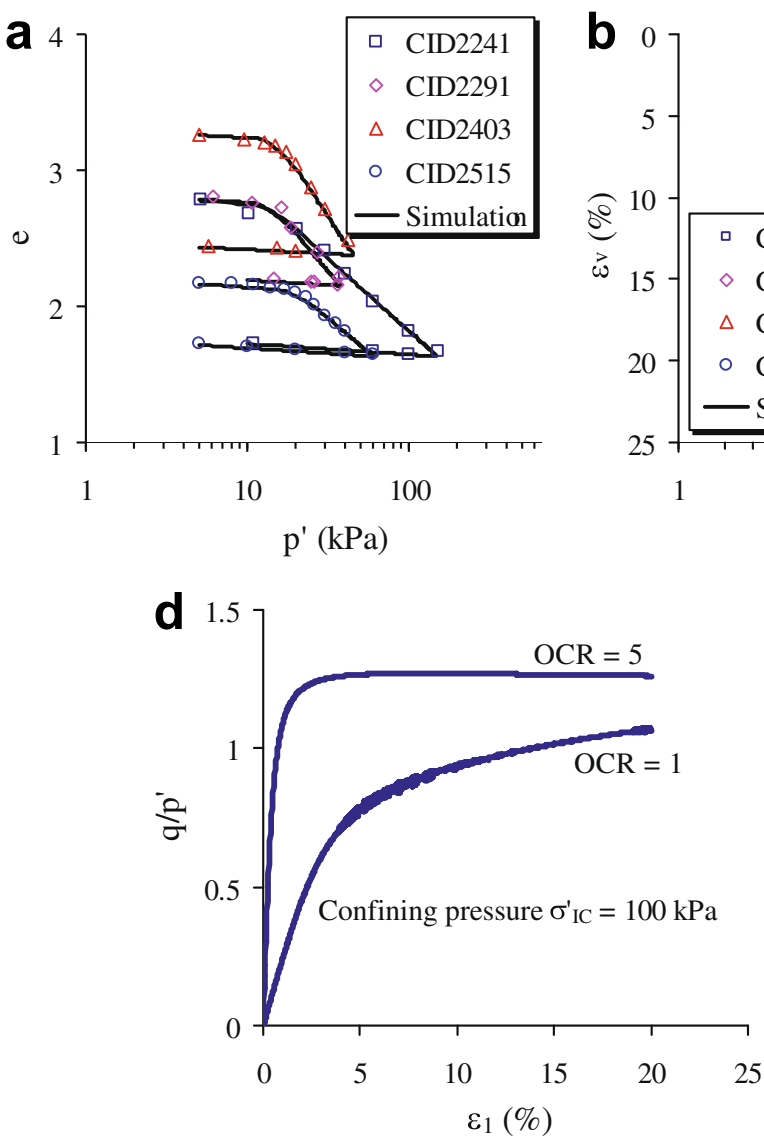
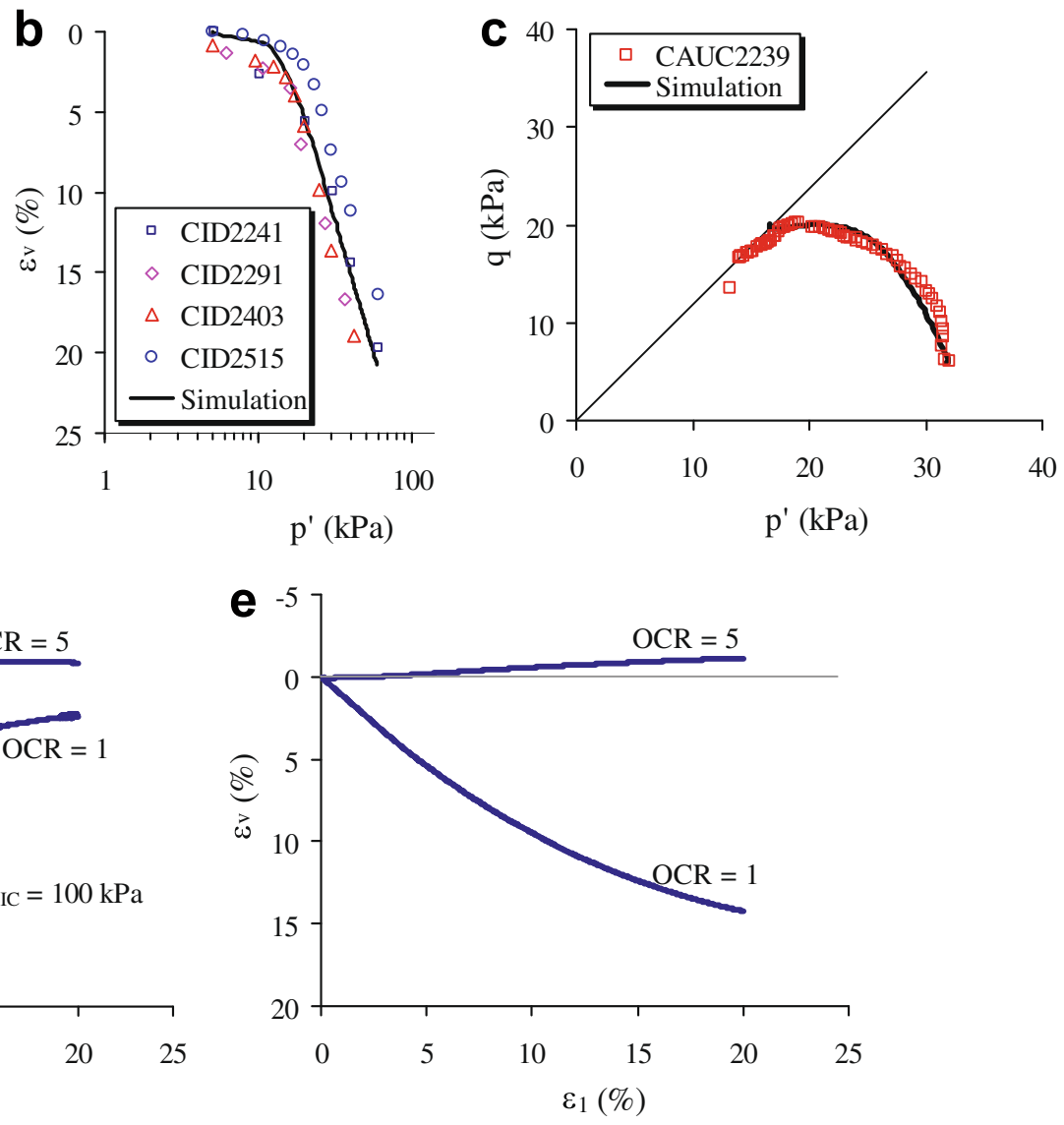

Fig. 4. Model prediction for tests: (a) using different parameters for each test, (b) using average parameters for all consolidation tests, (c) for undrained triaxial compression test, ( $d$ and e) for drained triaxial compression test.

Due to the large variation in the characteristics of the samples, three additional isotropic consolidation tests were selected to determine the model parameters by using the same procedure of calibration. All determined parameters and the averaged parameters are summarised in Table 3. The average parameters were used to simulate isotropic consolidation test and compared with all data from the four experimental results in Fig. 4b. The average parameters were also used to simulate the undrained triaxial compression tests and compared with experimental results as shown in Fig. 4c. The same set of parameters is also used to simulate drained triaxial compression tests for both normal and over consolidated Otaniemi clay as shown in Fig. 4d and e. Unfortunately, the experimental results on drained triaxial compression tests from Otaniemi clay at this field site are not available for comparison with the model simulation. However, the simulation appears to capture the main features of the drained triaxial compression behaviour for general clay. Thereby, the average values of the parameters seem to be suitable for representing the average properties of tested samples from Otaniemi clay.

\subsection{Test simulation and microstructural analysis}

In this section, three different cases of consolidation tests, namely $\eta_{1}=\eta_{0}, \eta_{2}<\eta_{1}$, and $\eta_{2}>\eta_{1}$ were analysed by means of the micromechanical approach, where $\eta_{0}, \eta_{1}$, and $\eta_{2}$ correspond to, respectively, the clay deposition, the first, and the second stages of loading. These tests are selected from Table 2.

The simulation of each test begins with a consolidation loading with $\eta_{0}=0.75$, which corresponds to the $K_{0}$ condition in the field. Along this stress path, the specimen is loaded to $p^{\prime}=15.7 \mathrm{kPa}$ and $q=11.75 \mathrm{kPa}$, which is equivalent to an effective overburden stress $23.5 \mathrm{kPa}$ at $4 \mathrm{~m}$ depth below ground. This process is used to simulate clay deposition in the field, as shown in the upper part of Fig. 5a, marked by points $a, b, c$. The corresponding points on a $e-\log p^{\prime}$ curve are shown in Fig. 5b. 

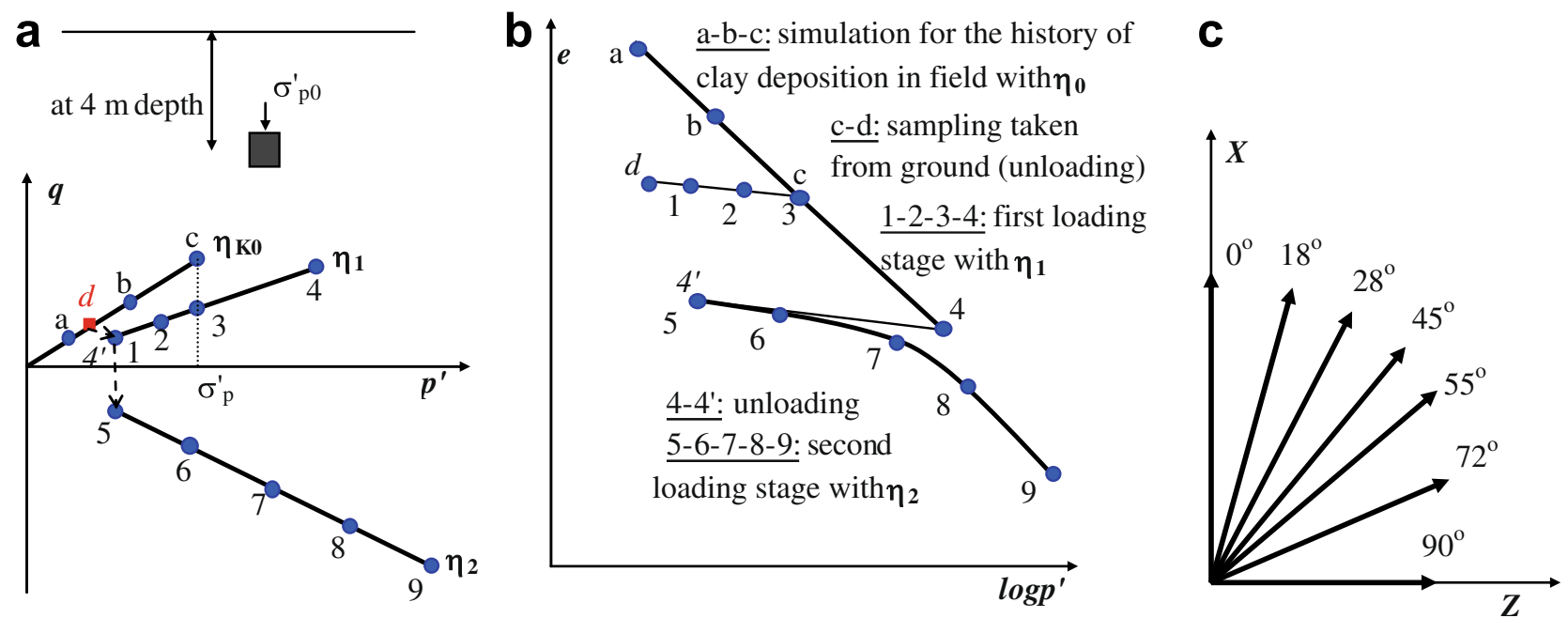

Fig. 5. (a and b) Simulation procedure and selected steps for rose diagram, and (c) five inter-particle orientations located on the $X-Z$ plane of the coordinate system.

Then, the specimen is unloaded to a very small stress value, along the path of $\eta=0.75$. This is a simulation of the sampling process, in which the sample is extracted from $4 \mathrm{~m}$ depth to the ground surface, as the rebound curve shown in Fig. $5 a$ and b, marked by $c, d$. Point 1 corresponds to the state of the specimen to be used in the laboratory for the first stage loading test.

The first stage loading is then simulated. The specimen is loaded with a constant $\eta_{1}$ to a pressure greater than the point of $p^{\prime}=15.7 \mathrm{kPa}$ and $q=11.75 \mathrm{kPa}$, as shown in Fig. $5 \mathrm{a}$ and b, marked by $1,2,3$, and 4 . The specimen is again unloaded to a very small value, along the path of $\eta_{1}$. The rebound curve is marked by points 4 and $4^{\prime}$.

Subsequently, the second stage loading is performed. The specimen is loaded with a constant $\eta_{2}$ to a pressure greater than that of point 4 (i.e., the ending point of the first loading stage), as shown in Fig. $5 \mathrm{a}$ and $\mathrm{b}$, marked by points $5,6,7,8$, and 9 . At this point, the simulation is completed.

For each test, the simulation results of $e-\log p^{\prime}$ curves are compared with the experimental data to evaluate the suitability of the model. The apparent yield points obtained from the experiments are also compared with those obtained from the predicted curves.

In addition, the local stress-strain behaviour at contact planes of various orientations is also plotted for analyses. The detailed plots for contact planes of various orientations will be described in a later section.

\subsubsection{Case 1: $\eta_{1}=\eta_{0}$}

Four consolidation tests (Series B in Table 2) along the $K_{0}$-consolidated stress path $\left(\eta_{1}=\eta_{0}=0.75\right)$ were simulated. After the clay deposit simulation along $\eta_{0}=0.75$, the first and second loading stages were then simulated. In this case we focus only on the results of the first loading stage, which are plotted in Fig. 6 and compared with experimental results. Good agreement between the experimental data and the simulation was achieved for the $\varepsilon_{v}-\log p^{\prime}$ curves. The apparent yield point can be obtained from a linear plot of $\varepsilon_{v}-p^{\prime}$ curve using a bilinear construction method as suggested by Mitchell (1970) and Karstunen and Koskinen (2008). The $\varepsilon_{v}-p^{\prime}$ plot is shown in Fig. 6b for both model simulation and experimental results. The yield points determined from both model simulation and experimental results are very close for this case.

The model simulation for the behaviour on the contact planes are also plotted to show the relations between the representative element and the different contact planes. Since the loading is symmetric around the $z$-axis, the orientation of a given contact plane can be defined by an inclined angle $\theta$ which is between the branch vector and the $z$-axis of the local coordinate system, as shown in Fig. $5 \mathrm{a}$. The angles $\theta$ selected are $0^{\circ}, 18^{\circ}, 28^{\circ}, 45^{\circ}, 55^{\circ}, 72^{\circ}$, and $90^{\circ}\left(\theta=0^{\circ}\right.$ corresponds to a horizontal plane $)$, as shown in the $x-z$ plane (Fig. $5 \mathrm{c}$ ).

Fig. 7 shows the local stress-strain relationships for the contact planes in the selected orientations. The local stress paths are plotted in the $\tau-\sigma$ plane, as shown in Fig. 7a, for both the clay deposition 

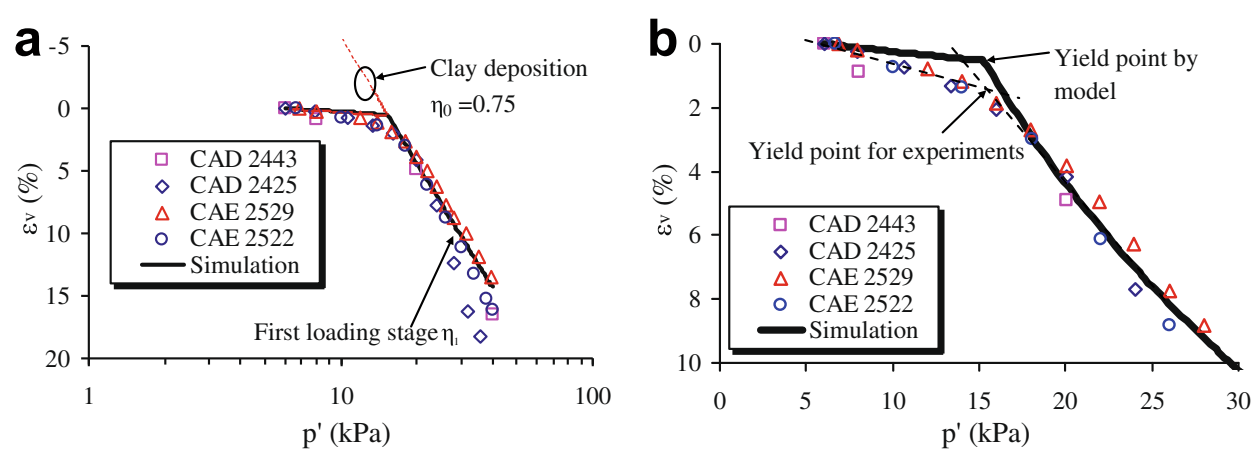

Fig. 6. Model prediction for tests with stress ratio $\eta$ the same as that of previous consolidation stage $\eta_{0}$.
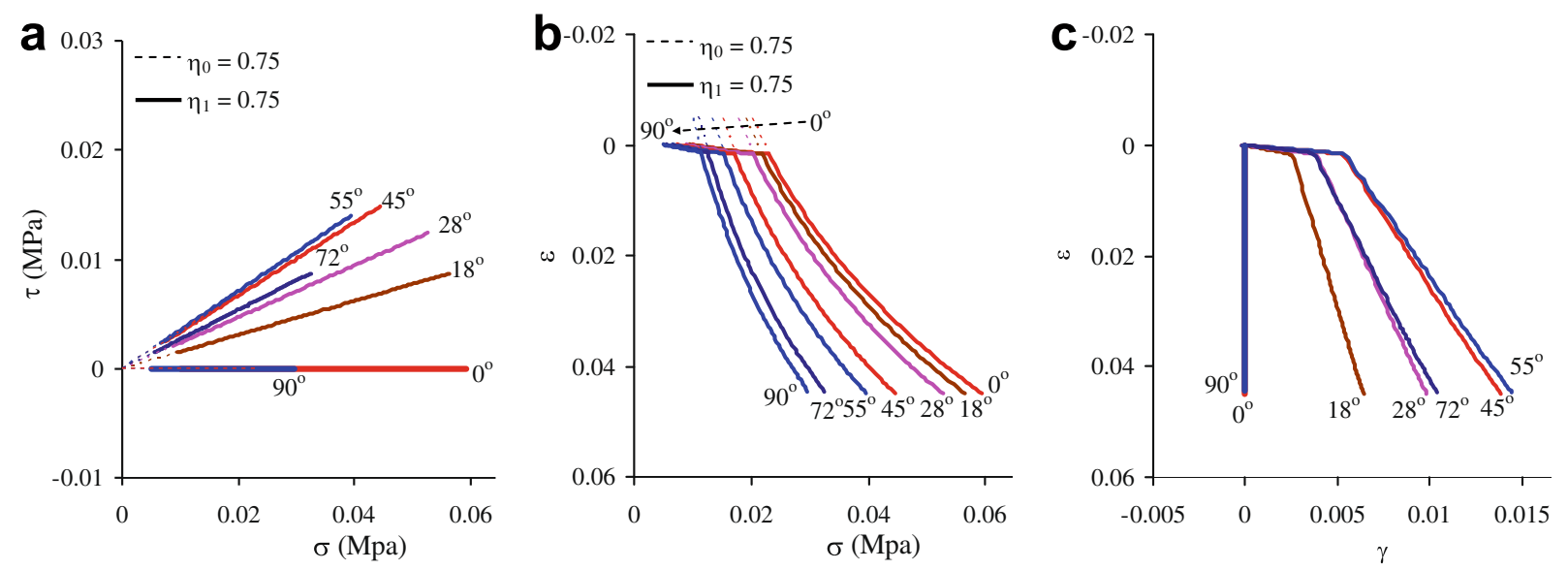

Fig. 7. Local behaviour on seven different inter-particle planes for the case $\eta=\eta_{1}$.

stage and the first loading stage. The local stress paths are different from one contact plane to another, under the load applied to the specimen. The shear component becomes more significant when the plane is more inclined. The maximum slope is near the planes oriented at $55^{\circ}$. No shear component is generated for the horizontal and vertical plane contacts $\left(0^{\circ}\right.$ and $\left.90^{\circ}\right)$. For the selected planes, the slope of the local stress path increases from plane orientation $0^{\circ}$ to $55^{\circ}$ and decreases from $55^{\circ}$ to $90^{\circ}$.

In the local normal stress-strain curves (see Fig. 7b), contact planes of all orientations yield simultaneously when the load $p$ applied to the specimen reaches its yield point (i.e., $15.7 \mathrm{kPa}$ as shown in Fig. $6 a$ and $b$ ). The elastic limits decrease from plane orientation $0^{\circ}$ to $90^{\circ}$. These elastic limits were created due to the previous load applied to the specimen during the clay deposition. In the local shear strain versus normal strain curves (see Fig. 7c), the amount of shear strain agrees with the slope of the local stress path, i.e., the larger slope leads to a larger shear strain.

In Fig. 8, the distribution of local stresses and strains versus plane orientations (in rose diagram) are plotted for the ending step $\mathrm{c}$ for the clay deposit stage and the selected steps $1,2,3$, and 4 for the first loading stage (see Fig. 5a and b).

It is noted that the normal stress $\sigma$ and shear stress $\tau$ distributions due to the clay deposition show a difference in all orientations, thus creating induced anisotropy of this material. This may lead to an irrecoverable microstructure alteration, thus producing and the inherent anisotropy.

The distributions expand in size from step 1 to 4 while keeping the same shape (see Fig. $8 a$ and $b$ ). Originally, the distribution of normal stress $\sigma$ is a point representing zero stresses in all directions. At the end of the clay deposition (step $c$ in Fig. 5a and b), the distribution expands to the location of the bold line plotted in Fig. 8a. This location represents the pre-consolidation pressures of all contact planes. During the sampling (c, $d$ in Fig. 5a and b), the distribution shrinks to the point of origin as an unloading process. Then, it expands again during the first loading stage. At step 3, the distribution reaches the bold line. It is noted that, during steps 1,2 , and 3, all contact planes are elastic until the 


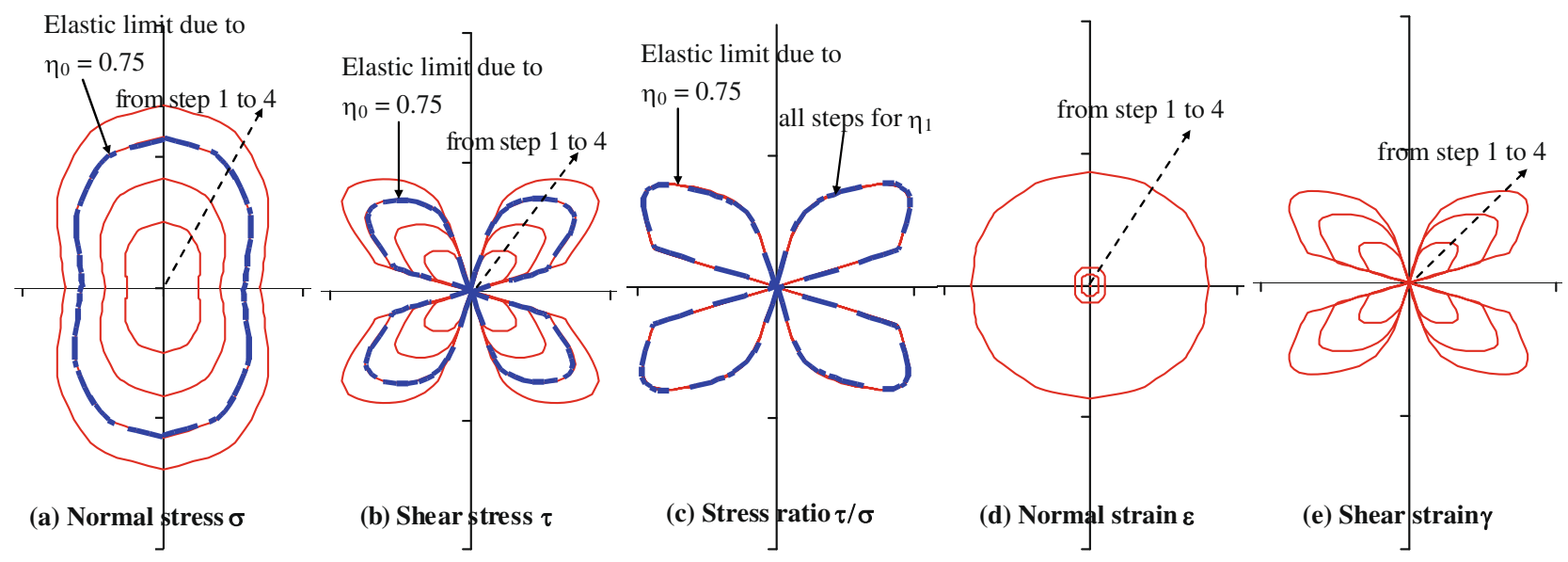

Fig. 8. Schematic plot for induced anisotropy for $\eta=0.75$.

distribution reaches the bold line. Then, all contact planes reach their pre-consolidation pressures simultaneously, and all planes begin to behave plastically, which can also be seen in Fig. 7b. At this point, the sample behaviour displays a sharp change in direction in the $\varepsilon_{v}-\log p^{\prime}$ as shown in Fig. 6a, representing the apparent yield point of the soil sample.

Fig. $8 \mathrm{c}$ shows the stress ratio at contact in all orientations. The elastic limits have not been exceeded in the first loading stage. Hence, small shear strains are expected.

Fig. 8d shows the distribution of normal strain, which implies slight differences in strains occurring for different plane orientations as shown in Fig. 7b. From step 3 to 4, the strain increases much higher than from step 2 to 3 due to the plastic strain occurring after the stresses reach the elastic limit. As for the distribution of shear strain in Fig. 8e, the shear strain from steps 3 to 4 does not show much difference in magnitude change from step 2 to 3, because the shear stresses are still in the elastic range.

\subsubsection{Case 2: $\eta_{2}<\eta_{1}$}

Three selected consolidation tests (Series B in Table 2) after an identical $\eta_{1}=0.75, \eta_{2}$ takes three different paths, $0.51,0.26$, and -0.52 as shown in Fig. 9. Simulation for each test includes sequentially the clay deposition $\eta_{0}=0.75$, the first stage loading $\eta_{1}=0.75$ and the second stage loading $\eta_{2}$. Since $\eta_{1}=\eta_{0}=0.75$ has already been presented in case 1 , we focus here on the behaviour during the second loading stage. General agreement between the experimental data and simulations was achieved for the $\varepsilon_{v}-\log p^{\prime}$ curves, as shown in Fig. 10a. Unlike case 1, the curve does not follow a bilinear pattern; instead, a smooth transition zone was found on the $\varepsilon_{v}-\log p^{\prime}$ plane at the location of the pre-consolidation stress (see Fig. 10a). The apparent yield point can be determined from the plots by using a bilinear construction method, as indicated in the previous case (see Fig. 10c and d). Although the yield points determined from simulation are in good agreement with the experiments, the curves from experiments show a wider scatter which can be attributed to the variability of the sample is initial void ratio while the prediction was made based on an averaged set of parameters.

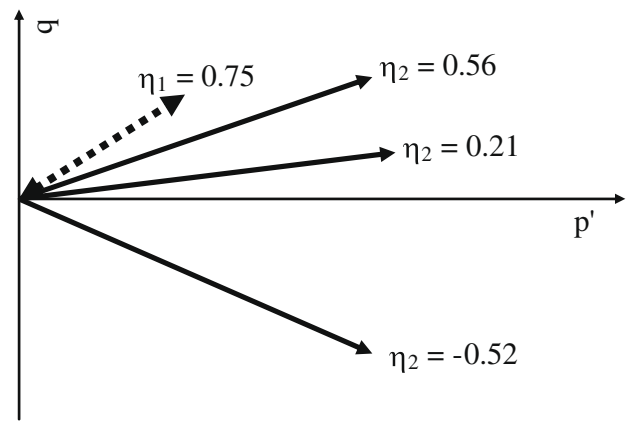

Fig. 9. Schematic plot of drained constant $\eta$ tests for $\eta_{2}<\eta_{1}$. 

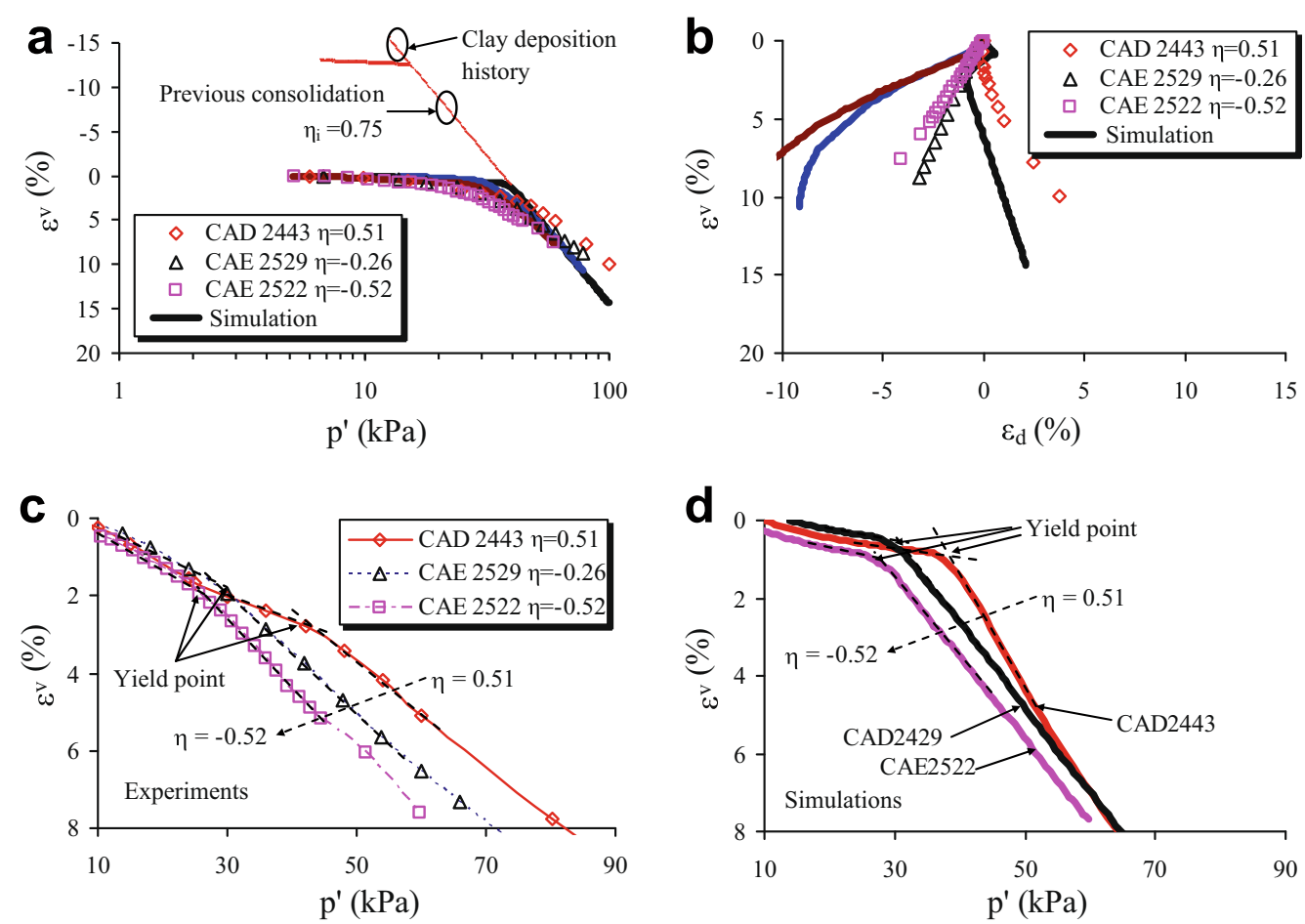

Fig. 10. Model prediction for tests with stress ratio $\eta$ smaller than that of previous consolidation stage $\eta_{1}$.
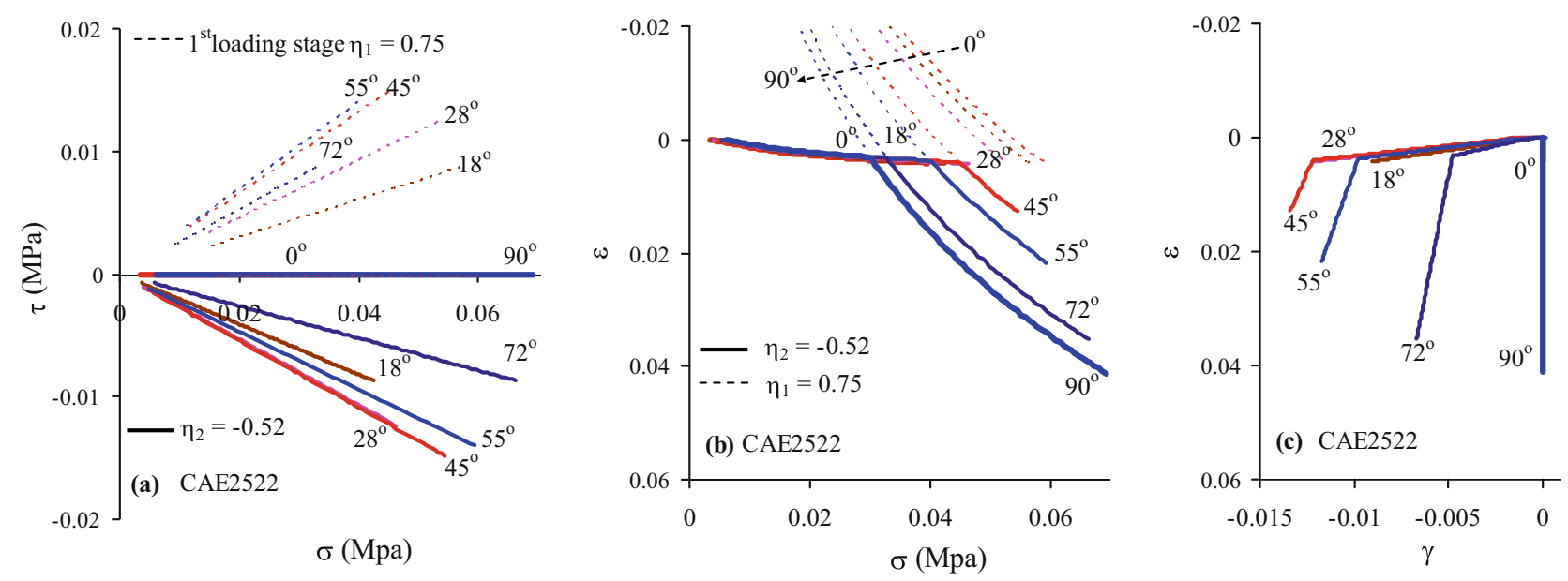

Fig. 11. Local behaviour on seven different inter-particle planes for the case $\eta_{2}<\eta_{1}$.

Among the three tests, $\eta_{2}=-0.52$ was selected in order to study the response on the contact planes. Fig. 11 shows the local stress-strain relationships for the contact planes in the selected orientations. The local stress paths are plotted in the $\tau-\sigma$ plane as shown in Fig. 11a for both the first and the second loading stage. The highest slope of the local stress paths is obtained for planes oriented at $55^{\circ}$ for the first loading stage and is for planes oriented at $45^{\circ}$ for the second loading stage. For the second loading stage, the slope of the local stress path increases from plane orientation $0^{\circ}$ to $45^{\circ}$ and decreases from $45^{\circ}$ to $90^{\circ}$.

In the local normal stress-strain curves (see Fig. 11b), only planes with orientation from $45^{\circ}$ to $90^{\circ}$ yielded. The planes with orientations from $0^{\circ}$ to $45^{\circ}$ did not yield even at the end of the second loading stage. Fig. 11c shows the local shear strain versus normal strain curves, the magnitude of local shear strain is greater for the stress paths with higher slopes on the $\sigma-\tau$ plane.

Fig. 12 shows the distribution of local stresses and strains in rose diagrams for the ending step 4 of the first loading stage and the selected steps 5, 6, 7, 8, and 9 for the second loading stage (see Fig. 5a 


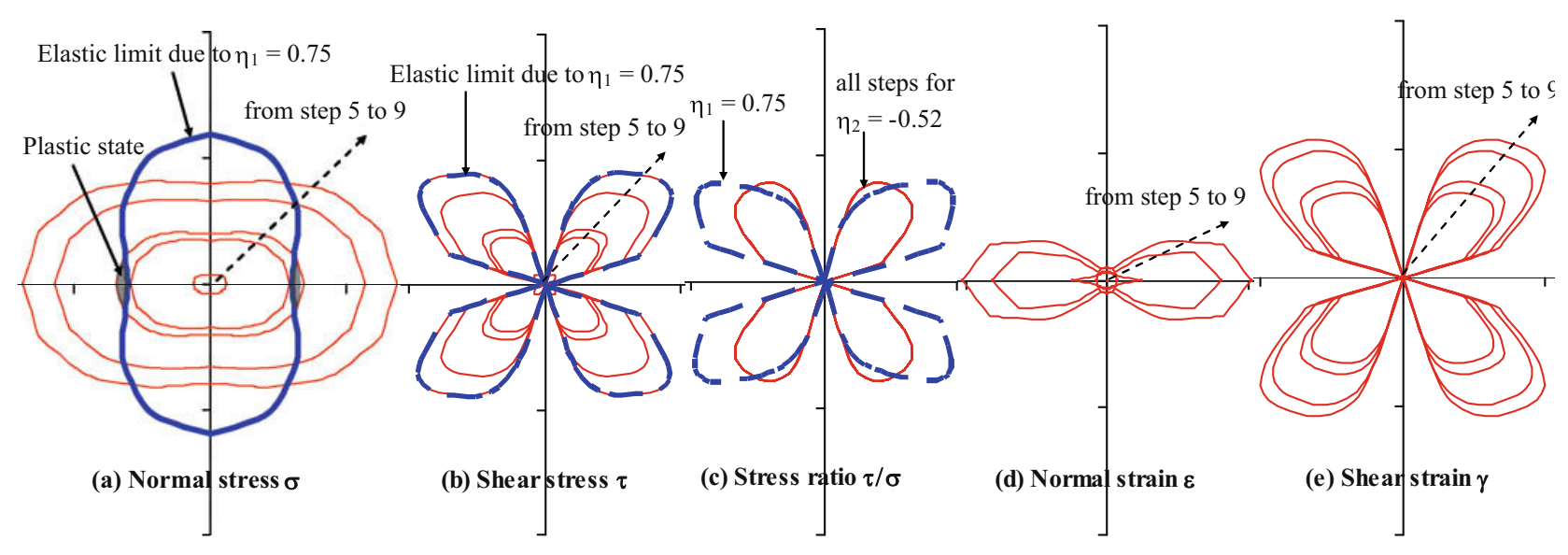

Fig. 12. Schematic plot for induced anisotropy for $\eta_{2}=-0.52$.

and $\mathrm{b})$. It is noted that step 4 gives the pre-stresses on each contact plane at the end of first stage loading.

The distribution of normal stress at the end of the first loading stage (step 4) has a long axis in the vertical direction. During the sampling ( $4-4^{\prime}$ in Fig. 5a and b), the distribution shrinks to the point of origin as an unloading process. Then, it expands again during the second loading stage from step 5 to 9, but with a different shape that has the long axis in the horizontal direction (see Fig. 12a). The change in shape of the distribution is due to the load pattern, changing from compression to extension (from $\eta_{1}=0.75$ to $\eta_{2}=-0.52$ ).

At step 6 of the second stage, the distribution reaches the bold line only for planes with near-horizontal orientations. Only these planes, after reaching their pre-consolidation pressures, begin to behave plastically. At steps 7, 8, and 9 the number of planes that reached their pre-consolidation stress (i.e., went beyond the bold line) continues to increase. At step 9, there are still nearly half of the planes which behave elastically, which is consistent with Fig. 11b which shows that all the contact planes with less than $45^{\circ}$ orientation did not yield. Thus, the soil has a smooth transition zone when it begins to yield in the curve $\varepsilon_{v}-\log p^{\prime}$, as shown in Fig. 10a, making it more difficult to define the apparent yield point based solely on globally applied stresses. It demonstrates that in this case, the first contact plane yields long before the apparent yield point determined from the bilinear construction, while many contact planes are still in the elastic state after the apparent yield point.

This case also shows that, due to a change of the loading path direction, the shape of the normal stress distribution can rotate around its principal axis, which indicates that the induced anisotropy involves not only the degree of anisotropy but also the axis of anisotropy.

Fig. 12b shows the distribution of shear stress and Fig. 12c shows the distribution of shear to normal stress ratio $\tau / \sigma$, which governs the shear deformation. The distributions show that after the second loading stage, there are a small number of contact planes (below $45^{\circ}$ orientation) which exceed the elastic limits created by the first loading stage.

Fig. 12d shows the distribution of local normal strains which indicates very small strains for contact planes having an orientation below $45^{\circ}$. For orientations greater than $45^{\circ}$, however, the planes display large normal strains due to their exceeding of the local elastic limit at early steps during this loading stage. Fig. 12e shows the shear strain distribution for the steps $6,7,8$, and 9 of the second loading stage. The magnitude of shear strains is relatively small because most of the contacts are still in the elastic range at step 9.

\subsubsection{Case 3: $\eta_{2}>\eta_{1}$}

Three selected consolidation tests (Series $\mathrm{C}$ in Table 2 ) are considered in this case. After an identical first loading path $\eta_{1}=0.11$, three different second loading paths occurred are followed $\eta_{2}=0.36,0.58$, and 0.83 as shown in Fig. 13. Simulation for each test includes, sequentially, the clay deposition $\eta_{0}=0.75$, the first stage loading $\eta_{1}=0.11$ and the second stage loading $\eta_{2}$. Particular attention was given to the second loading stage. Fig. 14a shows the $\varepsilon_{v}-\log p^{\prime}$ curves, and Fig. 14b the $\varepsilon_{v}-\varepsilon_{d}$ plane. 


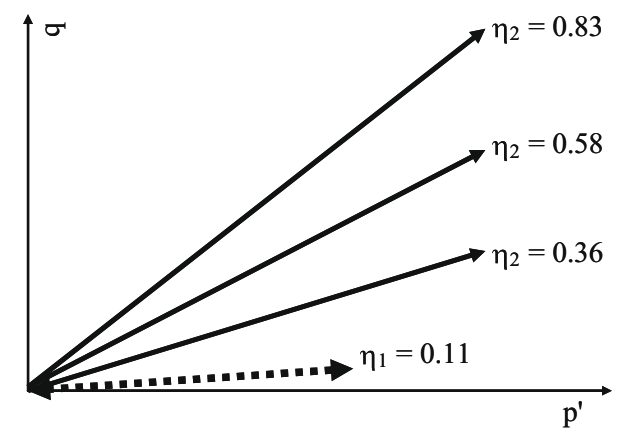

Fig. 13. Schematic plot of drained constant $\eta$ tests for $\eta_{2}>\eta_{1}$.
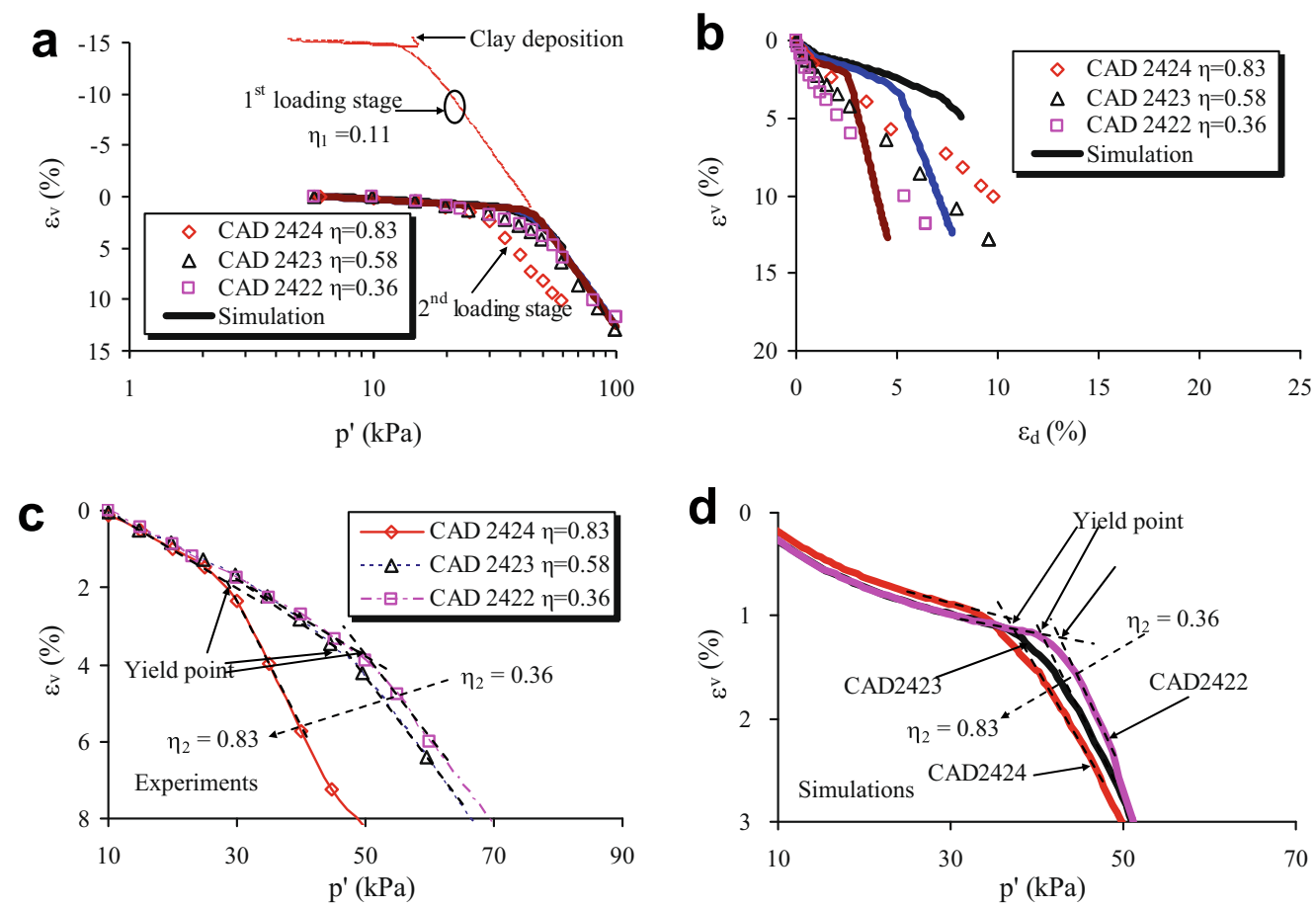

Fig. 14. Model prediction for tests with stress ratio $\eta$ bigger that of previous consolidation stage $\eta_{1}$.

Similar to case 2 , an obviously smooth transformation zone was found on the $\varepsilon_{v}-\log p^{\prime}$ plane, when the pre-consolidation stress was located. The apparent yield points determined from plots using a bilinear construction method are shown in the $\varepsilon_{v}-p^{\prime}$ plane in Fig. $14 \mathrm{c}$ and $\mathrm{d}$.

Among the three tests, $\eta_{2}=0.83$ was selected for studying the contact planes. Fig. 15 shows the local stress-strain relationships for the contact planes in the selected orientations. In Fig. 15a, the $\tau-\sigma$ curves are plotted for both the first and the second loading stages. The slopes of the $\tau-\sigma$ curves for the second loading stage $\left(\eta_{2}=0.83\right)$ are much higher than those for the first loading stage $\left(\eta_{1}=0.11\right)$.

In the local normal stress-strain curves, at the second loading stage (Fig. 15b), the elastic limits were reached for all planes except for the plane oriented at $90^{\circ}$. The magnitude of the normal stress decreases with the orientation angle caused by the stress distribution. In the local shear strain versus normal strain curves (Fig. 15c), the magnitude of local shear strain is greater for stress paths with higher slopes in the $\sigma-\tau$ plane. It is also noticeable that the shear strain in this case is larger than in the previous two cases.

Fig. 16 shows the distribution of local stress and strain in rose diagrams for step 4 of the first loading stage and the selected steps 5, 6, 7, 8, and 9 for the second loading stage (Fig. 5a and b). The bold 

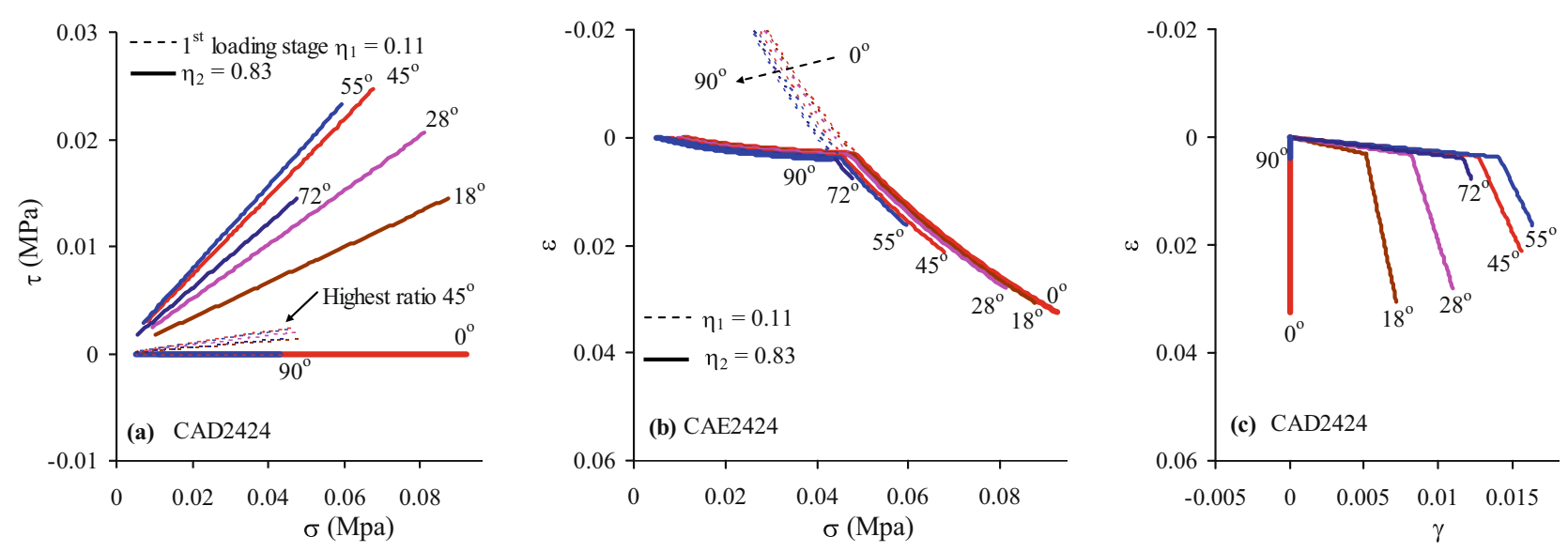

Fig. 15. Local behaviour on seven different inter-particle planes for the case $\eta_{2}>\eta_{1}$.
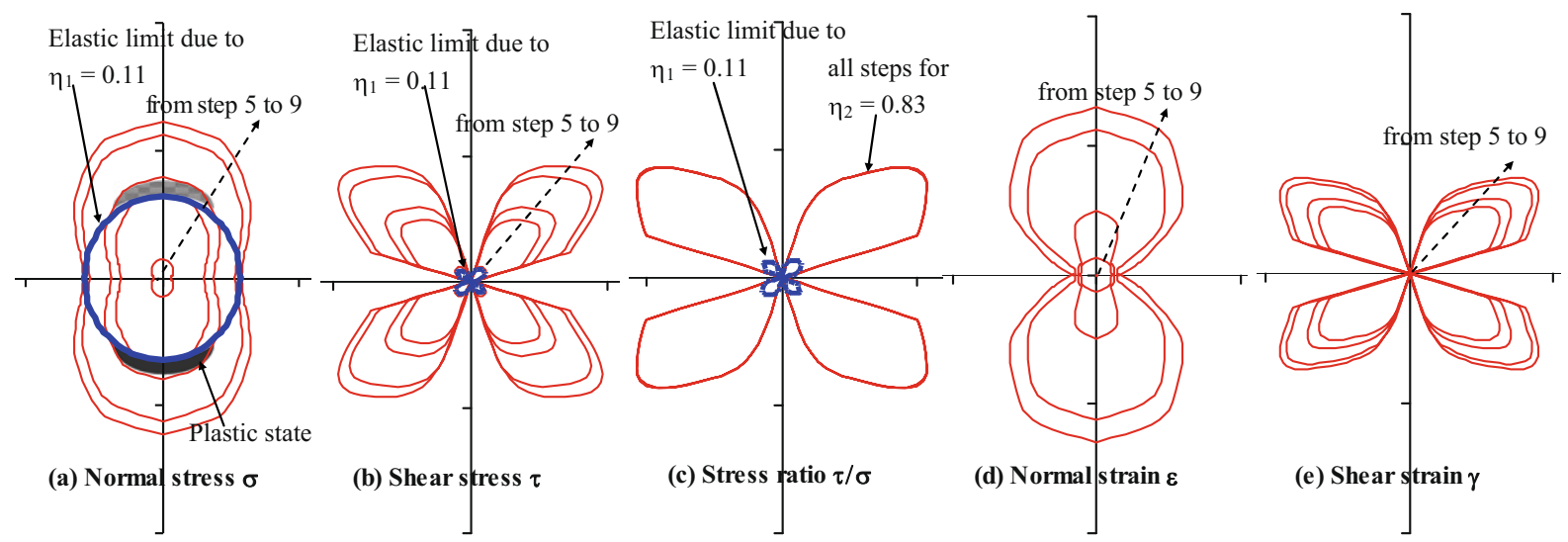

Fig. 16. Schematic plot for induced anisotropy for $\eta_{2}=0.83$.

line shows the stresses at step 4 at the end of the first loading stage, which also represents the elastic limits for the second loading stage.

Corresponding to the near isotropic consolidation of the first loading stage $\left(\eta_{1}=0.11\right)$, the bold line has a shape close to a circle with its long axis in the vertical direction. The distribution of the second loading stage $\left(\eta_{2}=0.83\right)$, however, has a shape much elongated in the vertical direction (Fig. 16a). When distribution starts to expand from origin during the second loading stage, it reaches the bold line (elastic limits) at first for planes with near-vertical orientations at step 6 . At steps 7, 8, and 9, the distribution expands further beyond the bold line. At the end of step 9, all planes yield, except for the planes with orientations near $90^{\circ}$, which is consistent with Fig. $15 \mathrm{~b}$ showing that all contacts yield except for the one with a $90^{\circ}$ orientation. The yielding process is also stretched over several load steps, thus the soil has a smooth transition zone when it begins to yield in the $\varepsilon_{v}-\log p^{\prime}$, as shown in Fig. 14a. It can be concluded that for the case of changing stress paths (stress path with various successive slopes $\eta$ ), the yielding condition does not occur simultaneously for all contact planes. Therefore, determining the yielding point from the test results at the macroscopic level becomes more difficult. This case also confirms that loading with reorientation of the stress path induces a change in the degree of anisotropy as well as in the direction of the anisotropy axis.

The fact that yielding condition does not occur simultaneously for all contact planes is a fundamental phenomenon for granular material. In fact, the result can be used to explain the incrementally nonlinear character of the material. As pointed out by many authors (Hill, 1965, 1966, 1967; Zienkiewicz and Pande, 1977; Bazant and Gambarova, 1984; Darve and Nicot, 2005), the mechanical state (elastic or plastic regime) of each contact depends on both the direction of the macroscopic loading and the orientation of the contact considered. As a consequence, the overall response corresponds to the 
contribution of the individual response of all the contact directions. A very complex, nonlinear and anisotropic behaviour is therefore obtained.

Fig. 16b shows the distribution of shear stress and Fig. 16c shows the distribution of shear to normal stress ratio $\tau / \sigma$. The distributions show that after the second loading stage, all planes exceed their shear elastic limits.

Fig. 16d shows the distribution of local normal strains which indicates very small strains for contact planes with near-horizontal orientation. Contact planes with near-vertical orientations, however, display large normal strains due to their stresses beyond the initial elastic limits. Fig. 16e shows the shear strain distribution for steps $6,7,8$, and 9 of the second loading stage. The magnitude of shear strains is relatively larger than in the previous two cases (see Fig. 15c) because the shear elastic limit is exceeded in contact planes of all orientations.

\subsection{Investigating the macro apparent yield curve}

As described in the previous section, the yield point is only an approximate description of the stress state, which serves as the elastic/plastic boundary for the material. Since the material cannot change its behaviour from elastic to plastic abruptly (i.e., contact planes are likely to yield in a sequential process), the yield point can be only approximately defined. The method used for determining the approximate yield point is a bilinear construction. In this section, the same method is used to construct the yield curve in a stress plane, as well as its kinematic rule based on the simulation of triaxial drained tests with different $\eta$-stress paths for the Otaniemi natural clay.

\subsubsection{Initial apparent yield curve}

In order to investigate the apparent yield curve of Otaniemi natural clay under clay deposition, drained tests of all series were simulated using the calibrated model parameters. Figs. 17 and 18 show the comparison between experimental results and model predictions for the full range of test stages for Series A and B-C, respectively. A general agreement was achieved for all test simulations compared to experiments. The under-prediction of volumetric strains during the first loading stage at high values of $\eta$ (e.g., CAD2260, CAD2464, CAD2261), as noted by Wheeler et al. (2003), can be attributed to the breakage of bonds among the clay clusters, which is not considered in the present microstructural model.

The apparent pre-consolidation pressures were measured by the bilinear method for all stress paths of the first loading stage. The initial apparent yield curve of Otaniemi natural clay in the normally consolidated region was then obtained, as shown in Fig. 19a. Considering the sample variability, the experimental data are scattered around the predicted results. The yield curve constructed from the results of the numerical simulations is approximately an elliptical shaped curve with a highest value of the mean effective stress lying approximately on the line $\eta=0.75$.

\subsubsection{Kinematic hardening of the macro apparent yield curve}

As investigated in the previous section, a change of stress history $\left(\eta_{2} \neq \eta_{1}\right)$ would redistribute the local stresses and strains, resulting in a change of the anisotropy axes. In a conventional plasticity model, the change of the anisotropic axes would be reflected by a change of the yield surface shape, and would require a kinematic hardening rule to model such behaviour.

Drained tests with different $\eta$ stress histories were simulated to construct the kinematic hardening of the yield curve. The $\eta$ value of the first loading stage varies from 0.98 to -0.34 , using tests of Series B for $\eta_{1}=0.75$ along natural deposition stress path, CAD2260 for $\eta_{1}=0.98$, Series C for $\eta_{1}=0.11$, CID2515 for $\eta_{1}=0$ and CAE2496 for $\eta_{1}=-0.34$. All tests were simulated by the microstructural model, as shown in Figs. 17 and 18. General agreement was achieved for the experimental and numerical results.

Fig. 19b-f shows the apparent yield surfaces for different consolidation histories described by the microstructural model. All yield curves have different shapes, which are approximately of elliptical type with a highest value of the mean effective stress lying approximately on the line of the $\eta_{1}$ stress path. In Fig. 19b, the stress path of the first loading stage is the same as that of the clay deposition (i.e., $\eta_{1}=\eta_{0}=0.75$ ). For this case, the yield surface moves and expands while keeping 

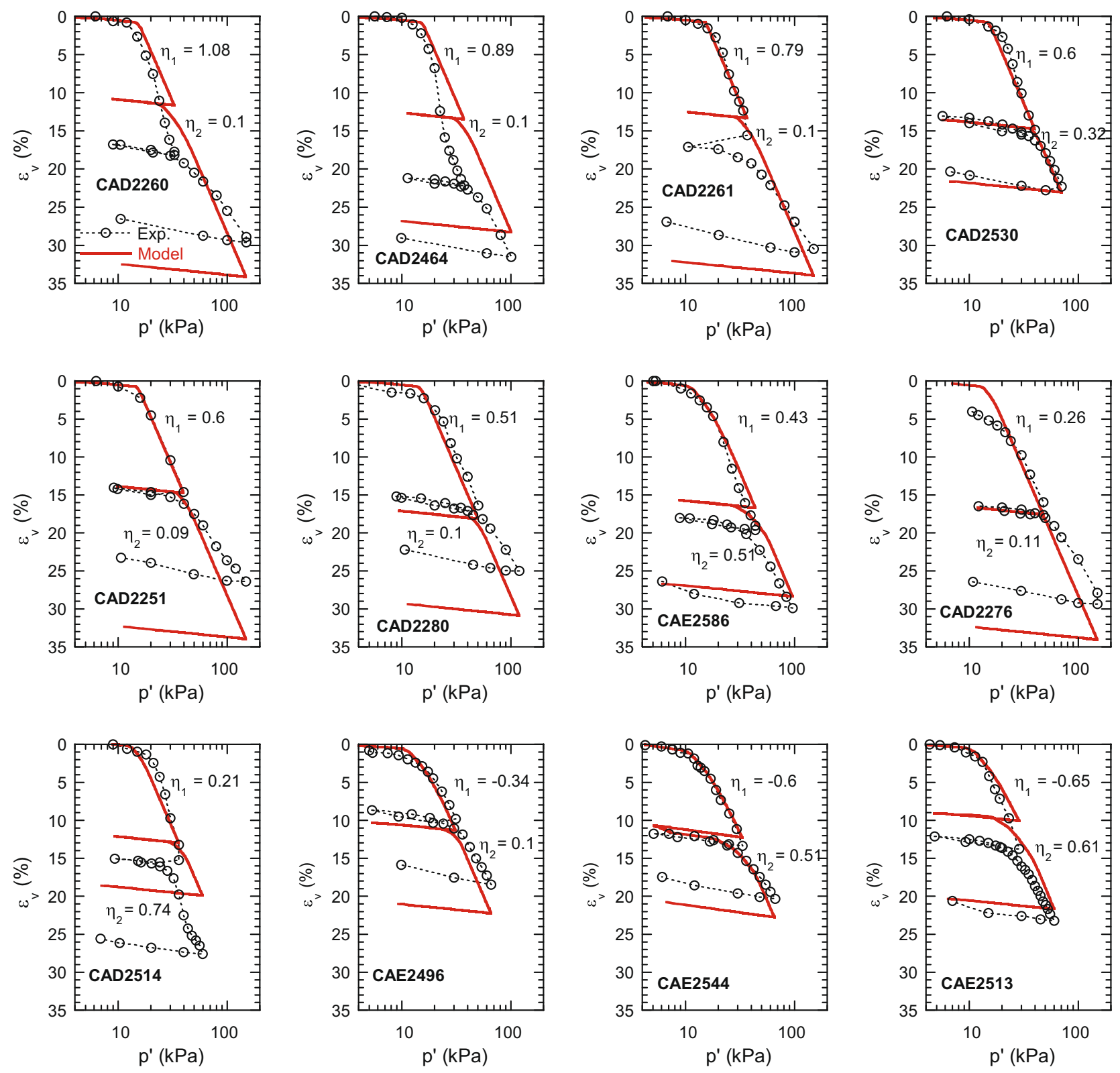

Fig. 17. Model predictions on drained tests Series A.

the same shape. Fig. $19 \mathrm{c}-\mathrm{f}$ shows cases with $\eta_{1} \neq \eta_{0}$. After the stress path $\eta_{1}$ is applied, the yield surface has not only moved but also changed its shape. The change in shape depends on the value of $\eta_{1}$. The data plotted in Fig. 19b-f, corresponding to the final yield surface, are obtained from the second loading stage in the test series. The constructed yield surfaces are compared to experimental results, when available, as shown in Fig. 19a-f. Considering the soil variability, the comparison shows a reasonably good agreement. The micromechanical approach seems capable of describing adequately the kinematic hardening of a yield curve in the stress space.

\section{Summary and conclusion}

A microstructural model for clay based on the approach proposed by Chang and Hicher (2005) has been used to simulate the multistage drained constant - $\eta$ tests on Otaniemi clay. The purpose of this study was to investigate the induced anisotropy due to various combinations of stress paths. It was attempted to link the mechanisms at inter-cluster contacts to the apparent 

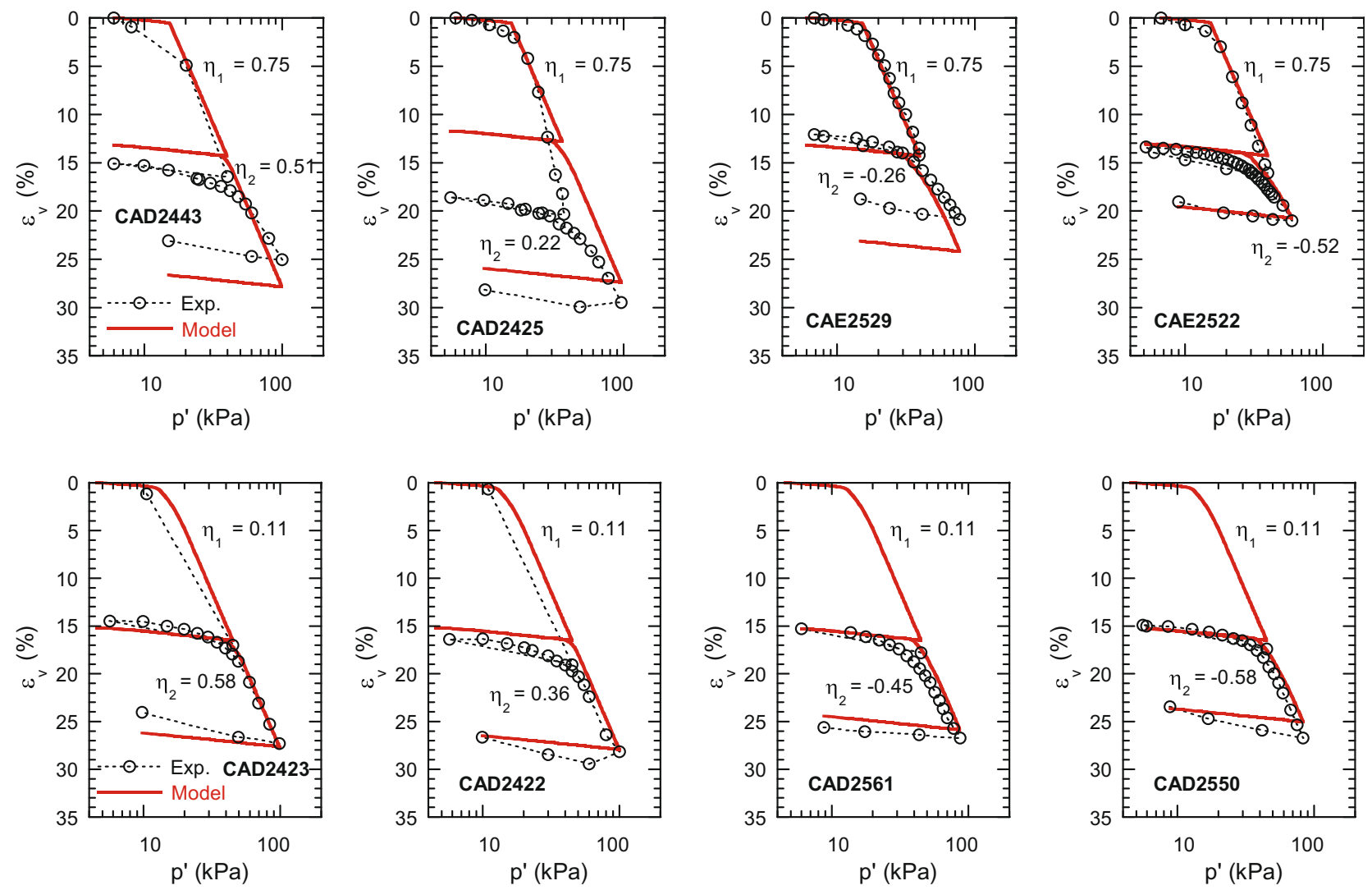

Fig. 18. Model predictions on drained tests Series B and C.

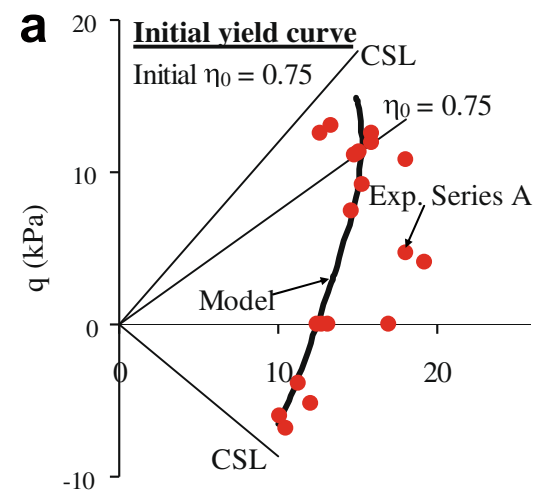

$\mathrm{p}^{\prime}(\mathrm{kPa})$

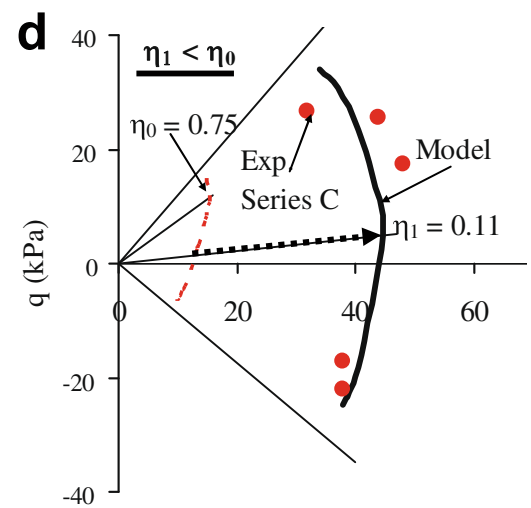

$\mathrm{p}^{\prime}(\mathrm{kPa})$

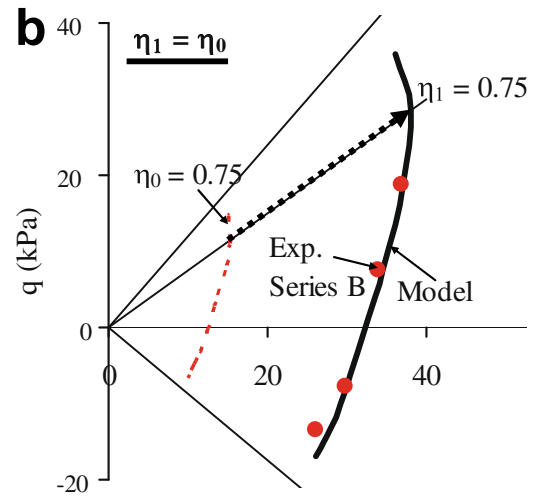

$\mathrm{p}^{\prime}(\mathrm{kPa})$

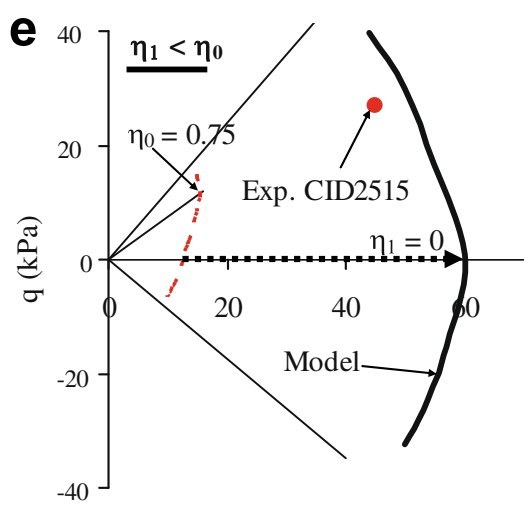

$\mathrm{p}^{\prime}(\mathrm{kPa})$

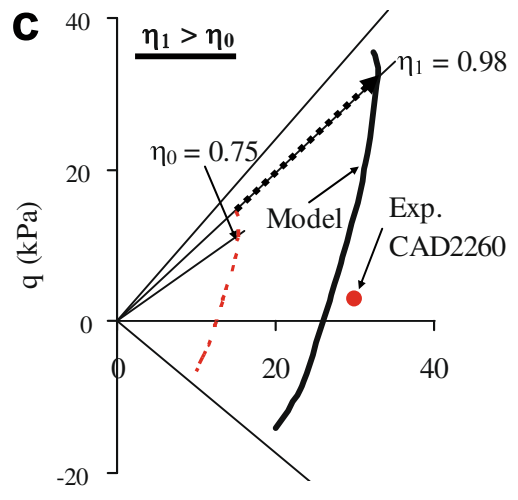

$\mathrm{p}^{\prime}(\mathrm{kPa})$

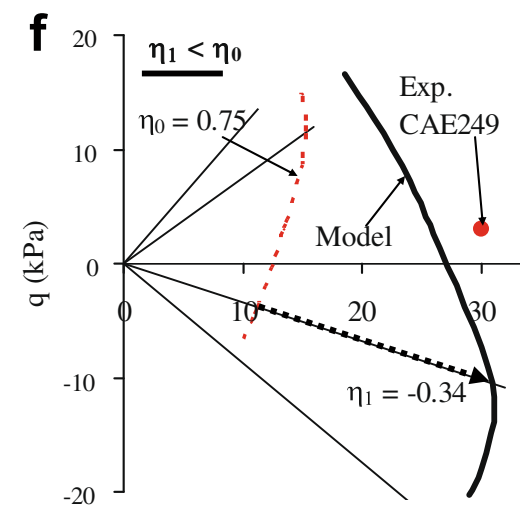

$\mathrm{p}^{\prime}(\mathrm{kPa})$

Fig. 19. Initial apparent yield curve and apparent yield curves for different consolidation history. 
yield surface and its kinematic hardening in the stress space. Three different cases have been studied:

(1) For the case $\eta_{1}=\eta_{0}$, the $e-\log p^{\prime}$ curve for the first loading stage is bilinear and the yield point is obviously situated at the intersection point of the two lines. When the applied stress reaches the pre-consolidation stress, all contact planes reach their pre-consolidation pressures simultaneously, and therefore they all begin to behave plastically.

(2) For the cases $\eta_{2}<\eta_{1}$ and $\eta_{2}>\eta_{1}$, the $e-\log p^{\prime}$ curve for the second loading stage is no longer bilinear, instead there is a smooth transition zone. In this case, the apparent yield point is determined by the bilinear construction method proposed by Mitchell (1970). When the applied stress reaches the pre-consolidation stress, not all contact planes reach their pre-consolidation pressures simultaneously. The number of yield contacts increases with applied load but some contacts are likely to remain elastic. The cause of this phenomenon is easily detected from the evolution of the local stress distribution for contacts of various orientations.

It can be concluded that for the case of stress paths with various successive directions, the yielding condition does not occur simultaneously for all contact planes. Therefore, the definition of yield becomes vague for the traditional plasticity theory, where the yield point occurs at a given applied stress.

It can also be concluded that, due to a change of loading path, the principal axis of the contact stress distribution can rotate, which indicates that the induced anisotropy includes not only the degree of anisotropy but also the principal axes of anisotropy.

Under the microstructural approach, the evolution of the state variables (local stress and strain) in the planes of all orientations is tracked. This leads to an account of anisotropy on stress-dependent properties, and can produce naturally the anisotropic behaviour without specifying a kinematic hardening yield surface in the stress space.

Given the good agreement between the numerical simulation and the experimental results, the micromechanical approach seems capable of modelling adequately the induced anisotropic behaviour of Otaniemi clay.

\section{Acknowledgements}

The work presented here was sponsored in part by the Academy of Finland (Grant 210744) and carried out as part of a Marie Curie Research Training Network "Advanced Modelling of Ground Improvement on Soft Soils (AMGISS)" supported by the European Community through the programme "Human Resources and Mobility". The writers would like to thank Matti Lojander at the Laboratory of Soil Mechanics and Foundation Engineering of Helsinki University of Technology, for his support.

\section{References}

Batdorf, S.B., Budianski, B., 1949. A mathematical theory of plasticity based on concept of slip. NACA Tech Note TN 1871.

Bazant, Z.P., Gambarova, P.G., 1984. Crack shear in concrete: crack band microplane model. ASCE Journal of Structural Engineering 110 (9), 2015-2035.

Bazant, Z.P., Xiang, Y., Ozbolt, J., 1995. Nonlocal microplane model for damage due to cracking. Proceedings of Engineering Mechanics 2, 694-697.

Biarez, J., Hicher, P.Y., 1994. Elementary Mechanics of Soil Behaviour. Balkema. pp. 208.

Burland, J.B., 1990. On the compressibility and shear strength of natural clays. Geotechnique 40 (3), 329-378.

Calladine, C.R., 1971. Microstructural view of the mechanical properties of saturated clay. Geotechnique 21, 391-415.

Chang, C.S., Gao, J., 1995. Second-gradient constitutive theory for granular material with random packing structure. International Journal of Solids and Structures 32 (16), 2279-2293.

Chang, C.S., Hicher, P.Y., 2005. An elastic-plastic model for granular materials with microstructural consideration. International Journal of Solids and Structures 42, 4258-4277.

Chang, C.S., Liao, C., 1990. Constitutive relations for particulate medium with the effect of particle rotation. International Journal of Solids and Structures 26, 437-453.

Chang, C.S., Sundaram, S.S., Misra, A., 1989. Initial moduli of particulate mass with frictional contacts. International Journal for Numerical and Analytical Methods in Geomechanics 13 (6), 626-641.

Christofferson, J., Mehrabadi, M.M., Nemat-Nassar, S., 1981. A micromechanical description on granular material behaviour. ASME Journal of Applied Mechanics 48, 339-344. 
Cudny, M., Vermeer, P.A., 2004. On the modelling of anisotropy and destructuration of soft clays within the multi-laminate framework. Computers and Geotechnics 31 (1), 1-22.

Dafalias, Y.F., 1986. An anisotropic critical state soil plasticity model. Mechanics Research Communications 13 (6), $341-347$.

Dafalias, Y.F., Manzari, M.T., Papadimitriou, A.G., 2006. SANICLAY: simple anisotropic clay plasticity model. International Journal for Numerical and Analytical Methods in Geomechanics 30 (12), 1231-1257.

Darve, F., Nicot, F., 2005. On incremental non-linearity in granular media: henomenological and multi-scale views (Part I). International Journal for Numerical and Analytical Methods in Geomechanics 29, 1387-1409.

Diaz Rodriguez, J.A., Leroueil, S., Aleman, J.D., 1992. Yielding of Mexico City clay and other natural clays. Journal of Geotechnical Engineering 118 (7), 981-995.

Hashiguchi, K., Mase, T., 2007. Extended yield condition of soils with tensile yield strength and rotational hardening. International Journal of Plasticity 23, 1939-1956.

Hashiguchi, K., 2008. Verification of compatibility of isotropic consolidation characteristics of soils to multiplicative decomposition of deformation gradient. Soils and Foundations 48, 597-602.

Hicher, P.Y., Wahyudi, H., Tessier, D., 2000. Microstructural analysis of inherent and induced anisotropy in clay. Mechanics of Cohesive-Frictional Materials 5 (5), 341-371.

Hill, R., 1965. Continuum micro-mechanics of elastoplastic polycrystals. Journal of the Mechanics and Physics of Solids 13, 89101.

Hill, R., 1966. Generalized relations for incremental deformation of metal crystals by multislip. Journal of the Mechanics and Physics of Solids 14 (2), 95-102.

Hill, R., 1967. On the classical constitutive relations for elastic-plastic solids. In: Broberg, B., Hult, J., Niordson, F. (Eds.), Recent Progress in Applied Mechanics, Folke Odqvist Volume. Almqvist and Wiksell, pp. 241-249.

Karstunen, M., Koskinen, M., 2004. Undrained shearing of soft natural clays. In: Proceedings of NUMOG IX, Ottawa, Canada, pp. 173-179.

Karstunen, M., Koskinen, M., 2008. Plastic anisotropy of soft reconstituted clays. Canadian Geotechnical Journal 45 (3), $314-328$.

Lai, Y., Jin, L., Chang, X., 2008. Yield criterion and elasto-plastic damage constitutive model for frozen sandy soil. International Journal of Plasticity. doi:10.1016/j.ijplas.2008.06.010.

Liao, C.L., Chang, T.P., Young, D., Chang, C.S., 1997. Stress-strain relationship for granular materials bases on hypothesis of best fit. International Journal of Solids and Structures 34 (31-32), 4087-4100.

Messerklinger, S., Kahr, G., Plötze, M., Giudici-Trausch, J., Springman, S.M., Lojander, M., 2003. Mineralogical and mechanical behaviour of soft Finnish and Swiss clays. In: Vermeer, P.A. et al. (Eds.), Proceedings of the International Workshop on Geotechnics of Soft Soils-Theory and Practice, Noordwijkerhout, The Netherlands, VGE, Essen, Germany, pp. 467-472.

Mindlin, R.D., 1969. Microstructure in linear elasticity. Archive for Rational Mechanics and Analysis 16, 51-78.

Mitchell, R.J., 1970. On the yielding and mechanical strength of Leda clays. Canadian Geotechnical Engineering 7, $297-312$.

Muir Wood, D., 1990. Soil Behaviour and Critical State Soil Mechanics. Cambridge University Press, Cambridge, UK.

Muraleetharan, K.K., Liu, C., Wei, C., Kibbey, T.C.G., Chen, L., 2008. An elastoplatic framework for coupling hydraulic and mechanical behavior of unsaturated soils. International Journal of Plasticity. doi:10.1016/j.ijplas.2008.04.001.

Nicot, F., Darve, F., 2007. Micro-mechanical bases of some salient constitutive features of granular materials. International Journal of Solids and Structures 44, 7420-7443.

Nova, R., 1985. Mathematical modelling of anisotropy of clays. In: Proceedings of the 11th ICSMFE, San Francisco, vol. 1, pp. 607-661.

Oda, M., 1977. Co-ordination number and its relation to shear strength of granular material. Soils and Foundations 17 (2), $29-$ 42.

Pestana, J.M., Whittle, A.J., 1999. Formulation of a unified constitutive model for clays and sands. International Journal for Numerical and Analytical Methods in Geomechanics 23, 1215-1243.

Pande, G.N., Sharma, K.G., 1982. Multi-laminate model of clays-a numerical evaluation of the influence of rotation of the principal stress axis. In: Desai, C.S., Saxena, S.K. (Eds.), Proceedings of Symposium on Implementation of Computer Procedures and Stress-Strain Laws in Geotechnical Engineering, Chicago. Acorn Press, Durham, NC, pp. 575-590.

Rothenburg, L., Selvadurai, A.P.S., 1981. Micromechanical definitions of the Cauchy stress tensor for particular media. In: Selvadurai, A.P.S. (Ed.), Mechanics of Structured Media, Amsterdam, pp. 469-486.

Tavenas, F., Lereoueil, S., 1977. Effects of stresses and time on yielding of clays. In: Proceedings of the Ninth ICSMFE 1, pp. 319326.

Tsutsumi, S., Kaneko, K., 2008. Constitutive response of idealized granular media under the principal stress axes rotation. International Journal of Plasticity 24 (11), 1967-1989.

Wheeler, S.J., Näätänen, A., Karstunen, M., Lojander, M., 2003. An anisotropic elasto-plastic model for soft clays. Canadian Geotechnical Journal 40, 403-418.

Whittle, A.J., Kavvadas, M.J., 1994. Formulation of MIT-E3 constitutive model for overconsolidated clays. Journal of Geotechnical Engineering 120 (1), 173-198.

Yang, Y., Muraleetharan, K.K., Yu, H.S., 2006. A middle surface concept model for saturated sands in general stress space. International Journal for Numerical and Analytical Methods in Geomechanics 30 (5), 389-412.

Zienkiewicz, O.C., Pande, G.N., 1977. Time dependent multilaminate model of rocks, a numerical study of deformation and failure of rock masses. International Journal for Numerical and Analytical Methods in Geomechanics 1, $219-247$. 